\title{
Inelastic electron-nucleus scattering and scaling at high inelasticity
}

\author{
M. B. Barbaro, ${ }^{1}$ J. A. Caballero, ${ }^{2}$ T. W. Donnelly, ${ }^{3}$ and C. Maieron ${ }^{2,4}$ \\ ${ }^{1}$ Dipartimento di Fisica Teorica, Università di Torino and INFN, Sezione di Torino, Via P. Giuria 1, 10125 Torino, Italy \\ ${ }^{2}$ Departamento de Física Atómica, Molecular y Nuclear, Universidad de Sevilla, Apartado Postal 1065, E-41080 Sevilla, Spain \\ ${ }^{3}$ Center for Theoretical Physics, Laboratory for Nuclear Science and Department of Physics, Massachusetts Institute of Technology, \\ Cambridge, Massachusetts 02139, USA \\ ${ }^{4}$ INFN, Sezione di Catania, Via S. Sofia 64, 95123 Catania, Italy
}

(Received 24 November 2003; published 24 March 2004)

\begin{abstract}
Highly inelastic electron scattering is analyzed within the context of the unified relativistic approach previously considered in the case of quasielastic kinematics. Inelastic relativistic Fermi gas modeling that includes the complete inelastic spectrum-resonant, nonresonant, and deep inelastic scattering-is elaborated and compared with experimental data. A phenomenological extension of the model based on direct fits to data is also introduced. Within both models, cross sections and response functions are evaluated and binding energy effects are analyzed. Finally, an investigation of the second-kind scaling behavior is also presented.
\end{abstract}

DOI: 10.1103/PhysRevC.69.035502

PACS number(s): 25.30.Fj, 24.10.Jv, 13.60.Hb

\section{INTRODUCTION}

In this work we consider highly inelastic electron scattering and compare its analysis with the case of quasielastic (QE) electron scattering. The latter is dominated by the process where the exchanged virtual photon interacts with a nucleon in the nuclear ground state and ejects that nucleon, thereby forming a nuclear particle-hole excitation. Corrections to this dominant process involve going beyond the impulse approximation to account for two-body currents, finalstate interactions, and nuclear correlations. Although these contributions are known not to be entirely negligible [1-7] this simple process accounts for the basic feature seen in the vicinity of elastic scattering from a nucleon at rest, namely, the QE peak. Models such as those discussed below take into account the fact that the nucleons in the nucleus are moving and are bound and thereby produce a broad peak in the inelastic spectrum. In the present work our goal is to extend the analysis, still maintaining the same basic features of the relativistic modeling used for the QE region, and now focus on what we call highly inelastic scattering, or for brevity, simply the inelastic region. This includes everything that goes beyond the $\mathrm{QE}$ process: that is, whereas the $\mathrm{QE}$ process assumes elastic scattering from the nucleons, the inelastic process will assume inelastic $e-N$ scattering. For relatively low final-state invariant masses one lies in the region of resonance excitation and two cases of this sort have been explored in recent work $[8,9]$. In the present study these ideas are generalized to include the complete inelastic spectrum, both resonant and nonresonant, including deep inelastic scattering (DIS), within the context of the unified relativistic approach used in our previous work.

Thus, in the present work our goal is to begin by exploring extensions of the relativistic Fermi gas (RFG) model $[6,10,11]$ to an inelastic version of this approach. While this bears some connection with traditional convolution models for the high-energy response of nuclei (see, for example, Refs. [12-15]) it is not the same in that, albeit within a model, it correctly incorporates a specific relativistic nuclear spectral function into the problem, whereas some other ap- proaches make additional assumptions and use only the integral of the spectral function, namely, the nuclear momentum distribution or make nonrelativistic approximations when dealing with the spectral function.

This distinction can be seen quite clearly in studies of first- and second-kind scaling [11,16-20] and will not be elaborated here. Once the inelastic RFG modeling is in hand, it becomes clear that it might be useful to explore a phenomenological extension of this model, namely, what we call the extended relativistic Fermi gas (ERFG). In this approach we take the result of doing the correct integral over the nuclear spectral function (i.e., not the full integral, which is the momentum distribution, as alluded to above) directly from fits made previously to the data [21]. We shall see that this has a significant impact on the nuclear responses at high inelasticity.

An issue which will also become clear later is that the story is not yet complete: in addition to the modeling done in the present work, where the focus is placed on incorporating inelastic effects at high energies, there are still other contributions that must be added. Specifically, in recent work [22] on $2 p-2 h$ meson-exchange current effects it is seen that a significant incoherent contribution must be added to those explored here. Given that the work on $2 p-2 h$ effects is, as yet, incomplete-correlation contributions are presently being included-it is premature to make too much of comparisons with experimental data, and, as we remark later in the appropriate places, the final understanding of how all of the various reaction mechanisms enter, while becoming clearer is not yet achieved.

The paper is organized as follows: in Sec. II we recall the general formalism for inelastic electron-nucleus scattering; in Sec. III we derive the expressions for the inelastic hadronic tensor in three different models: the pure RFG model [Sec. III A], the RFG including the effects of binding energy [Sec. III B] and the ERFG [Sec. III C]; in Sec. IV we present numerical results for cross sections [Sec. IV A], response functions [Sec. IV B], and scaling functions [Sec. IV C] and finally, in Sec. V we draw our conclusions. 


\section{INELASTIC ELECTRON-NUCLEUS SCATTERING: GENERAL FORMALISM}

With the goal outlined above in mind, we start by rewriting the general expressions that apply in both elastic and inelastic regimes. The general formalism describing inclusive electron-nucleus scattering processes is widely available [23-25]; here we simply focus on those aspects that are of special relevance for the discussion that follows. We follow the conventions and metric of Ref. [26] and use capital letters to refer to four-vectors. The incident and scattered electron four-momenta are denoted by $K_{i}^{\mu}=\left(\varepsilon_{i}, \mathbf{k}_{i}\right)$ and $K_{f}^{\mu}$ $=\left(\varepsilon_{f}, \mathbf{k}_{f}\right)$. The hadronic variables, $P_{A}^{\mu}=\left(M_{A}, \mathbf{0}\right)$ and $P_{B}^{\mu}$ $=\left(E_{B}, \mathbf{p}_{B}\right)$ represent the four momenta of the target and residual nucleus, respectively. The four-momentum transfer is given by $Q^{\mu}=(\omega, \mathbf{q})$ (we assume the Born approximation, i.e., only one virtual photon exchanged in the process).

Following standard procedures the differential cross section may be written

$$
\frac{d \sigma}{d \Omega_{f} d \varepsilon_{f}}=\frac{2 \alpha^{2}}{Q^{4}} \frac{\varepsilon_{f}}{\varepsilon_{i}} \eta_{\mu \nu} W^{\mu \nu},
$$

where $\alpha$ is the fine structure constant, $\eta_{\mu \nu}$ is the leptonic tensor that can be evaluated directly using trace techniques [27], and $W^{\mu \nu}$ is the hadronic tensor containing all of the nuclear structure and dynamics information. Assuming that the final state can be described in terms of a recoiling nuclear state $\left|\psi_{B}\right\rangle$ plus a (highly) inelastic state $\left|\Phi_{X}\right\rangle$, its general expression is given by

$$
\begin{aligned}
W^{\mu \nu}= & \overline{\sum_{A}} \sum_{B} \sum_{X}\left\langle\psi_{B}, \Phi_{X}\left|\hat{J}^{\mu}(\mathbf{q})\right| \psi_{A}\right\rangle^{*}\left\langle\psi_{B}, \Phi_{X}\left|\hat{J}^{\nu}(\mathbf{q})\right| \psi_{A}\right\rangle \\
& \times \rho\left(E_{B}\right) d E_{B} \rho\left(E_{X}\right) d E_{X} \delta\left(\varepsilon_{i}-\varepsilon_{f}+E_{A}-E_{B}-E_{X}\right),
\end{aligned}
$$

where $\bar{\Sigma}_{A}\left(\Sigma_{B} \Sigma_{X}\right)$ indicates the appropriate average (sum) over initial (final) states. Here $\hat{J}^{\mu}(\mathbf{q})$ is the Fourier transform of the nuclear current operator evaluated, $\left|\psi_{A}\right\rangle$ and $\left|\psi_{B}, \Phi_{X}\right\rangle$ represent the initial and final states, respectively, and the distribution functions $\rho\left(E_{B}\right)$ and $\rho\left(E_{X}\right)$ are introduced to account for the energy-momentum dispersion relation of the final nuclear $(B)$ and hadronic $(X)$ systems. In this work we assume that the inelasticity of the process is totally accounted for by the final state $\Phi_{X}$; hence for the energy distribution function of the residual nuclear system we use $\rho\left(E_{B}\right)=\delta\left(E_{B}-\bar{E}_{B}\right)$, where $\bar{E}_{B}=\sqrt{\mathbf{p}_{B}^{2}+\left(M_{B}^{*}\right)^{2}}$. Note that $W^{\mu \nu}$ in Eq. (2) is meant to be evaluated at $\mathbf{p}_{B}+\mathbf{p}_{X}=\mathbf{q}=\mathbf{k}_{i}-\mathbf{k}_{f}$.

The nuclear tensor can equivalently be expressed as an integral in the $(\mathcal{E}, p)$ plane, with $-\mathbf{p}=\mathbf{p}_{B}$ the three-momentum of the recoiling daughter nucleus and $\mathcal{E} \equiv \sqrt{\mathbf{p}^{2}+\left(M_{B}^{*}\right)^{2}}$ $-\sqrt{\mathbf{p}^{2}+\left(M_{B}^{0}\right)^{2}}$ the excitation energy of the residual nucleus (see Ref. [19]). The domain of integration is the kinematically allowed region

$$
\max [\mathcal{E}(0), 0] \leqslant \mathcal{E} \leqslant \mathcal{E}(\pi),
$$

where

$$
\mathcal{E}(\theta)=M_{A}^{0}+\omega-\sqrt{\left(M_{B}^{0}\right)^{2}+p^{2}}-\sqrt{W_{X}^{2}+q^{2}+p^{2}+2 p q \cos \theta}
$$

with $\theta$ the angle between $\mathbf{p}$ and $\mathbf{q}$, and where $W_{X}$ is the invariant mass of the final state. In the $M_{B}^{0} \rightarrow \infty$ limit the above expression becomes

$$
\mathcal{E}_{\infty}(\theta)=m_{N}+\widetilde{\omega}-\sqrt{W_{X}^{2}+q^{2}+p^{2}+2 p q \cos \theta}
$$

where $m_{N}$ is the nucleon mass, $\widetilde{\omega} \equiv \omega-E_{S}$ and $E_{S}=M_{B}^{0}+m_{N}$ $-M_{A}^{0}$ is the separation energy.

The upper curve $\mathcal{E}(\pi)$ crosses the $p$ axis at $p_{-}=-y_{X}$ and $p_{+}=Y_{X}$, where

$$
\begin{aligned}
y_{X}= & \frac{1}{2 W^{2}}\left[\left(M_{A}^{0}+\omega\right) \sqrt{\left(W-M_{B}^{0}\right)^{2}-W_{X}^{2}}\right. \\
& \left.\times \sqrt{\left(W+M_{B}^{0}\right)^{2}-W_{X}^{2}}-2 q \Lambda_{X}\right]
\end{aligned}
$$

and

$$
\begin{aligned}
Y_{X}= & \frac{1}{2 W^{2}}\left[\left(M_{A}^{0}+\omega\right) \sqrt{\left(W-M_{B}^{0}\right)^{2}-W_{X}^{2}}\right. \\
& \left.\times \sqrt{\left(W+M_{B}^{0}\right)^{2}-W_{X}^{2}}+2 q \Lambda_{X}\right],
\end{aligned}
$$

and where

$$
W=\sqrt{\left(M_{A}^{0}+\omega\right)^{2}-q^{2}} \quad \text { and } \quad \Lambda_{X}=\frac{1}{2}\left[W^{2}+\left(M_{B}^{0}\right)^{2}-W_{X}^{2}\right] \text {. }
$$

The variable $y_{X}$ is the generalization of the usual $y$-scaling variable to the inelastic process where a resonance $X$ is produced. In the limit $M_{B}^{0} \rightarrow \infty$ it reads

$$
y_{X, \infty}=\sqrt{\left(\widetilde{\omega}+m_{N}\right)^{2}-W_{X}^{2}}-q .
$$

Note that the allowed region decreases with $W_{X}$ and collapses to a point when $-y_{X}=Y_{X}$, which implies $W=M_{B}^{0}+W_{X}$ or, in the $M_{B}^{0} \rightarrow \infty$ limit, $\left(y_{X, \infty}\right)_{\min }=-q$, corresponding to $\left(W_{X}\right)_{\max }=\widetilde{\omega}+m_{N}$. Summarizing, for fixed four-momentum transfer, the resonant mass is limited to the range

$$
m_{N}+m_{\pi} \leqslant W_{X} \leqslant m_{N}+\omega-E_{S} .
$$

\section{THE RELATIVISTIC FERMI GAS MODEL}

In this section we proceed by evaluating the hadronic nuclear tensor assuming the impulse approximation and by working within the framework of the RFG model. In this case, the virtual photon is absorbed by an on-shell nucleon described by a Dirac spinor $u\left(\mathbf{h}, s_{h}\right)$, with energy $\bar{E}_{\mathbf{h}}$ $=\sqrt{\mathbf{h}^{2}+m_{N}^{2}}$. Integrating over the momenta in the Fermi sea, the following expression for the inelastic hadronic tensor results: 


$$
\begin{aligned}
W^{\mu \nu}(\mathbf{q}, \omega)= & \frac{3 \mathcal{N}}{4 \pi p_{F}^{3}} \int_{F} d \mathbf{h} \frac{m_{N}}{\bar{E}_{\mathbf{h}}} \int d E_{X} \delta\left(\omega+\bar{E}_{\mathbf{h}}-E_{X}\right) \\
& \times \frac{1}{2} \sum_{s_{h}} \sum_{X_{i}} \rho\left(E_{X_{i}}\right)\left[\bar{\Phi}_{X_{i}} \hat{J}^{\mu} u\left(\mathbf{h}, s_{h}\right)\right]^{*}\left[\bar{\Phi}_{X_{i}} \hat{J}^{\nu} u\left(\mathbf{h}, s_{h}\right)\right],
\end{aligned}
$$

where $\mathcal{N}$ is the number of nucleons (protons or neutrons) and $\int_{F} d \mathbf{h} \equiv \int d \mathbf{h} \theta\left(p_{F}-h\right), p_{F}$ being the Fermi momentum. The symbol $\int d E_{X}$ stands for the integral over the energy of the inelastic final state, while $\Sigma_{X_{i}}$ indicates in general the sum/ integral over all the internal quantum numbers of all possible inelastic final states $\Phi_{X_{i}}$, having total energy $E_{X}$ and total momentum $\mathbf{p}_{X}$, fixed by momentum conservation to be $\mathbf{p}_{X}$ $=\mathbf{h}+\mathbf{q}$.

The hadronic tensor in Eq. (11) for inelastic processes can be also written in the form

$$
\begin{aligned}
W_{\text {inel }}^{\mu \nu}(\mathbf{q}, \omega)= & \frac{3 \mathcal{N}}{4 \pi p_{F}^{3}} \int d E_{X} \int_{F} d \mathbf{h} \frac{m_{N}}{\bar{E}_{\mathbf{h}}} w_{\text {inel }}^{\mu \nu}\left(H, Q, E_{X}\right) \\
& \times \delta\left(\omega+\bar{E}_{\mathbf{h}}-E_{X}\right),
\end{aligned}
$$

where $H^{\mu}=\left(\bar{E}_{\mathbf{h}}, \mathbf{h}\right)$ and we have introduced the inelastic single-nucleon tensor

$$
\begin{aligned}
w_{\text {inel }}^{\mu \nu}\left(H, Q, E_{X}\right)= & \frac{1}{2} \sum_{s_{h}} \sum_{X_{i}} \rho\left(E_{X_{i}}\right)\left[\bar{\Phi}_{X_{i}} \hat{J}^{\mu} u\left(\mathbf{h}, s_{h}\right)\right]^{*} \\
& \times\left[\bar{\Phi}_{X_{i}} \hat{J}^{\nu} u\left(\mathbf{h}, s_{h}\right)\right] .
\end{aligned}
$$

Note that the above single-nucleon tensor has dimensions of $E^{-1}$. As will be shown later, this is in contrast with our past work on $\mathrm{QE}$ and $N \rightarrow \Delta$ scattering where the single-nucleon tensors were defined to be dimensionless.

Next we choose to express the inelastic hadronic tensor in Eq. (12) in terms of the invariant mass $W_{X}$,

$$
\begin{aligned}
W_{\text {inel }}^{\mu \nu}(\mathbf{q}, \omega)= & \frac{3 \mathcal{N}}{4 \pi p_{F}^{3}} \int d W_{X} \int_{F} d \mathbf{h} \frac{m_{N} W_{X}}{\bar{E}_{\mathbf{h}} E_{X}} w_{i n e l}^{\mu \nu}\left(H, Q, E_{X}\right) \\
& \times \delta\left(\omega+\bar{E}_{\mathbf{h}}-E_{X}\right)
\end{aligned}
$$

with $E_{X}=\sqrt{\mathbf{p}_{X}^{2}+W_{X}^{2}}$. The energy integral can be performed by exploiting the $\delta$ function, yielding

$$
W_{i n e l}^{\mu \nu}(\mathbf{q}, \omega)=\frac{3 \mathcal{N}}{4 \pi p_{F}^{3}} \int_{F} d \mathbf{h} \frac{m_{N}}{\bar{E}_{\mathbf{h}}} w_{i n e l}^{\mu \nu}\left(H, Q, \omega+\bar{E}_{\mathbf{h}}\right) .
$$

In the case of DIS on a single nucleon, the inelastic tensor simply reduces to the single-nucleon tensor $w_{\text {inel }}^{\mu \nu}$.

Before entering into a detailed analysis of the inelastic nuclear tensor, it is interesting to notice how the usual expressions for the $\mathrm{QE}$ and $N \rightarrow \Delta$ hadronic tensors are recovered from the general result given in Eq. (11). First, in the case of QE scattering, the nuclear final state is simply a particle-hole excitation, hence, in the RFG model, $\Phi_{X}$ describes an on-shell nucleon, namely, $\Phi_{X}=\sqrt{m_{N} / \bar{E}_{\mathbf{p}}} u\left(\mathbf{p}, s_{p}\right)$. The energy distribution function is simply $\rho\left(E_{X}\right)=\delta\left(E_{X}\right.$
$-\bar{E}_{\mathbf{p}}$ ) and the sum over the final states reduces to a sum over spin projections, $\Sigma_{X_{i}}=\Sigma_{s_{p}}$. The QE hadronic tensor then reads

$$
W_{Q E}^{\mu \nu}(\mathbf{q}, \omega)=\frac{3 \mathcal{N}}{4 \pi p_{F}^{3}} \int_{F} d \mathbf{h} \frac{m_{N}^{2}}{\bar{E}_{\mathbf{h}} \bar{E}_{\mathbf{p}}} w_{Q E}^{\mu \nu}(H, Q) \delta\left(\omega+\bar{E}_{\mathbf{h}}-\bar{E}_{\mathbf{p}}\right),
$$

where $w_{Q E}^{\mu \nu}$ is the usual dimensionless $\mathrm{QE}$ single-nucleon tensor

$$
w_{Q E}^{\mu \nu}=\frac{1}{2} \sum_{s_{h}} \sum_{s_{p}}\left[\bar{u}\left(\mathbf{p}, s_{p}\right) \hat{J}^{\mu} u\left(\mathbf{h}, s_{h}\right)\right]^{*}\left[\bar{u}\left(\mathbf{p}, s_{p}\right) \hat{J}^{\nu} u\left(\mathbf{h}, s_{h}\right)\right] .
$$

In the case of the transition $N \rightarrow \Delta$, the final state $\Phi_{X}$, within the context of the RFG model, is an on-shell $\Delta$, namely, $\Phi_{X}=\sqrt{m_{\Delta} / \bar{E}_{\Delta}} u_{\Delta}\left(\mathbf{p}, s_{\Delta}\right)$, with on-shell energy $\bar{E}_{\Delta}=\sqrt{\mathbf{p}^{2}+m_{\Delta}^{2}}$. The energy distribution function in this case is $\rho\left(E_{X}\right)$ $=\delta\left(E_{X}-\bar{E}_{\Delta}\right)$ and $\Sigma_{X_{i}}=\Sigma_{s_{\Delta}}$. The $N \rightarrow \Delta$ hadronic tensor that results is

$$
W_{\Delta}^{\mu \nu}(\mathbf{q}, \omega)=\frac{3 \mathcal{N}}{4 \pi p_{F}^{3}} \int_{F} d \mathbf{h} \frac{m_{N}^{2}}{\bar{E}_{\mathbf{h}} \bar{E}_{\Delta}} w_{\Delta}^{\mu \nu}(H, Q) \delta\left(\omega+\bar{E}_{\mathbf{h}}-\bar{E}_{\Delta}\right)
$$

with $w_{\Delta}^{\mu \nu}$ the dimensionless nucleon- $\Delta$ tensor

$$
w_{\Delta}^{\mu \nu}=\frac{m_{\Delta}}{2 m_{N}} \sum_{s_{h}} \sum_{s_{\Delta}}\left[\bar{u}_{\Delta}\left(\mathbf{p}, s_{p}\right) \hat{J}^{\mu} u\left(\mathbf{h}, s_{h}\right)\right]^{*}\left[\bar{u}_{\Delta}\left(\mathbf{p}, s_{p}\right) \hat{J}^{\nu} u\left(\mathbf{h}, s_{h}\right)\right] .
$$

As expected, these expressions for the dimensionless singlenucleon tensors coincide with the ones introduced in Ref. $[8,28]$. Likewise for the Roper resonance the expressions obtained in Ref. [9] are recovered.

\section{A. The RFG inelastic nuclear tensor and response functions}

In this section we evaluate the inelastic nuclear tensor in the RFG framework. For convenience, as usual we first define the dimensionless variables

$$
\begin{gathered}
\boldsymbol{\kappa}^{\mu}=(\lambda, \boldsymbol{\kappa})=\left(\frac{\omega}{2 m_{N}}, \frac{\mathbf{q}}{2 m_{N}}\right), \quad \tau=\boldsymbol{\kappa}^{2}-\lambda^{2}, \\
\eta_{F}=\frac{p_{F}}{m_{N}}, \quad \epsilon_{F}=\sqrt{1+\eta_{F}^{2}}, \\
\eta^{\mu}=(\bar{\epsilon}, \boldsymbol{\eta})=\left(\frac{\bar{E}_{\mathbf{h}}}{m_{N}}, \frac{\mathbf{h}}{m_{N}}\right), \quad \mu_{X}=\frac{W_{X}}{m_{N}}, \\
\epsilon_{X}=\sqrt{\mu_{X}^{2}+(\boldsymbol{\eta}+2 \boldsymbol{\kappa})^{2}},
\end{gathered}
$$

in terms of which the hadronic tensor in Eq. (14) reads 


$$
\begin{aligned}
W_{\text {inel }}^{\mu \nu}(\kappa, \lambda)= & \frac{3 \mathcal{N}}{4 \pi \eta_{F}^{3}} \int d \mu_{X} \int d \boldsymbol{\eta} \frac{\mu_{X}}{\bar{\epsilon} \epsilon_{X}} w_{i n e l}^{\mu \nu}\left(\boldsymbol{\eta}, \mu_{X} ; \kappa, \lambda\right) \\
& \times \delta\left(2 \lambda+\bar{\epsilon}-\epsilon_{X}\right) \theta\left(\eta_{F}-\eta\right) .
\end{aligned}
$$

Before presenting the explicit results for the RFG response functions, let us discuss an important ingredient of the calculation, the single-nucleon inelastic hadronic tensor $w_{i n e l}^{\mu \nu}$. For unpolarized scattering, the latter can be parametrized in terms of two structure functions, $w_{1}$ and $w_{2}$, according to

$$
w_{\text {inel }}^{\mu \nu}=-w_{1}\left(g^{\mu \nu}+\frac{\kappa^{\mu} \kappa^{\nu}}{\tau}\right)+w_{2}\left(\eta^{\mu}+\kappa^{\mu} \rho\right)\left(\eta^{\nu}+\kappa^{\nu} \rho\right) .
$$

For on-shell nucleons, the structure functions $w_{1}$ and $w_{2}$ depend on two variables, the four-momentum transfer $Q^{2}$ and the invariant mass $W_{X}$ of the final state reached by the nucleon, or, equivalently, the single-nucleon Bjorken variable

$$
x=\frac{\left|Q^{2}\right|}{2 H \cdot Q}=\frac{\left|Q^{2}\right|}{W_{X}^{2}-m_{N}^{2}-Q^{2}}=\frac{\tau}{\eta \cdot \kappa} .
$$

In our formalism it is convenient to introduce the inelasticity parameter $[8,29]$

$$
\rho \equiv 1+\frac{1}{4 \tau}\left(\mu_{X}^{2}-1\right),
$$

the value unity corresponding to elastic scattering. Note that $\rho$ is simply linked to the Bjorken scaling variable of the on-shell nucleon moving inside the target nucleus by the relation $\rho=1 / x$, thus in the following we will use $\rho$ as argument of the structure functions $w_{1}, w_{2}$.

In presenting our results we will also use the "laboratory" Bjorken variable

$$
x_{L}=\frac{\left|Q^{2}\right|}{2 m_{N} \omega}=\frac{\tau}{\lambda},
$$

corresponding to a single nucleon at rest in the laboratory frame.

Let us now return to the inelastic nuclear tensor of Eq. (21): after performing the polar angular integration by means of the energy-conserving $\delta$ function one gets

$$
\begin{aligned}
W_{\text {inel }}^{\mu \nu}(\kappa, \lambda)= & \frac{3 \mathcal{N} \tau}{2 \eta_{F}^{3} \kappa} \int_{0}^{2 \pi} \frac{d \Phi}{2 \pi} \int_{\rho_{1}(\kappa, \lambda)}^{\rho_{2}(\kappa, \lambda)} d \rho \int_{\epsilon_{0}(\rho)}^{\epsilon_{F}} d \bar{\epsilon} \\
& \times w_{\text {inel }}^{\mu \nu}\left(\bar{\epsilon}, \theta_{0}, \rho ; \kappa, \lambda\right),
\end{aligned}
$$

where

$$
\cos \theta_{0}=\frac{1}{\kappa \eta}(\lambda \bar{\epsilon}-\tau \rho) .
$$

The condition $\left|\cos \theta_{0}\right| \leqslant 1$ fixes the integration limits over $\bar{\epsilon}$ :

$$
\bar{\epsilon} \geqslant \epsilon_{0}(\rho) \equiv \kappa \sqrt{\frac{1}{\tau}+\rho^{2}}-\lambda \rho .
$$

Moreover, by requiring that $\epsilon_{0}(\rho) \leqslant \epsilon_{F}$ and that the resonance mass is above the pion-production threshold (i.e., $\mu_{X}$ $\geqslant \mu_{\text {thresh }} \equiv 1+\mu_{\pi}$ ) the following region is obtained for the integration over $\rho$ :

$$
\left[\rho_{1}(\kappa, \lambda), \rho_{2}(\kappa, \lambda)\right]=\left[\max \left\{\frac{\lambda \epsilon_{F}-\kappa \eta_{F}}{\tau}, \rho_{\text {thresh }}\right\}, \frac{\lambda \epsilon_{F}+\kappa \eta_{F}}{\tau}\right]
$$

with

$$
\rho_{\text {thresh }}=1+\frac{\mu_{\pi}\left(\mu_{\pi}+2\right)}{4 \tau} .
$$

Note that the upper integration limit $\rho_{2}(\kappa, \lambda)$ always lies below the cutoff corresponding to Eq. (10). Indeed using Eqs. (10) and (24) and keeping in mind that the (negative) separation energy of the RFG is $E_{S}^{R F G}=-T_{F} \equiv m_{N}\left(1-\epsilon_{F}\right)$, the maximum $\rho$ allowed by Eq. (10) reads

$$
\rho_{\text {max }}^{R F G}=1+\frac{1}{4 \tau}\left[\left(2 \lambda+\epsilon_{F}\right)^{2}-1\right]=\rho_{2}(\kappa, \lambda)+\frac{1}{4 \tau}\left(2 \kappa-\eta_{F}\right)^{2},
$$

$\rho_{2}$ therefore resulting in the more stringent integration limit.

Now by writing the single-nucleon inelastic tensor in terms of structure functions $w_{1}$ and $w_{2}$ as in Eq. (22) and choosing the $z$ direction along $\mathbf{q}$, the integration over $\Phi$ and $\bar{\epsilon}$ can be performed analytically (see Appendix A) and the hadronic inelastic tensor can be expressed in the general form

$$
\begin{aligned}
W_{\text {inel }}^{\mu \nu}(\kappa, \lambda)= & \frac{3 \mathcal{N} \tau}{2 \eta_{F}^{3} \kappa} \xi_{F} \int_{\rho_{1}(\kappa, \lambda)}^{\rho_{2}(\kappa, \lambda)} d \rho\left(1-\psi_{X}^{2}\right) \theta\left(1-\psi_{X}^{2}\right) \\
& \times U^{\mu \nu}(\kappa, \tau, \rho),
\end{aligned}
$$

where $\xi_{F}=\epsilon_{F}-1$ is the Fermi kinetic energy and the inelastic scaling variable

$$
\psi_{X} \equiv \operatorname{sgn}(\lambda-\tau \rho) \sqrt{\frac{\epsilon_{0}(\rho)-1}{\epsilon_{F}-1}}
$$

has been defined. For each value of $\rho$ (and hence $\mu_{X}$ ) a "peak" can thus be identified, corresponding to the region $-1 \leqslant \psi_{X} \leqslant 1$, centered at

$$
\begin{gathered}
\psi_{X}=0, \quad \lambda_{P}=\tau_{P} \rho=\frac{1}{2 \rho}\left(\sqrt{1+4 \kappa_{P}^{2} \rho^{2}}-1\right), \\
\kappa_{P}=\sqrt{\tau_{P}\left(1+\tau_{P} \rho^{2}\right)},
\end{gathered}
$$

whose width

$$
\Delta \lambda=\frac{1}{2}\left[\sqrt{\left(2 \kappa+\eta_{F}\right)^{2}+\mu_{X}^{2}}-\sqrt{\left(2 \kappa-\eta_{F}\right)^{2}+\mu_{X}^{2}}\right] \simeq \frac{2 \kappa \eta_{F}}{\sqrt{4 \kappa^{2}+\mu_{X}^{2}}}
$$

is a function that grows with $\kappa$ and decreases with $\mu_{X}$. 
The general expression for the tensor $U^{\mu \nu}$ is derived in Appendix A. Here we only report the longitudinal and transverse components

$$
\begin{aligned}
U^{L}= & U^{00}=\frac{\kappa^{2}}{\tau}\left[\left(1+\tau \rho^{2}\right) w_{2}(\tau, \rho)-w_{1}(\tau, \rho)\right. \\
& \left.+w_{2}(\tau, \rho) \mathcal{D}(\kappa, \tau, \rho)\right], \\
U^{T}= & U^{11}+U^{22}=2 w_{1}(\tau, \rho)+w_{2}(\tau, \rho) \mathcal{D}(\kappa, \tau, \rho),
\end{aligned}
$$

which are linked to the longitudinal and transverse response functions by the following relations:

$$
\begin{aligned}
R_{\text {inel }}^{L, T}(\kappa, \tau)= & \frac{3 \mathcal{N} \tau}{2 \eta_{F}^{3} \kappa} \xi_{F} \int_{-1}^{\psi_{X}^{\max }(\kappa, \lambda)} d \psi_{X}\left|\frac{\partial \rho}{\partial \psi_{X}}\right|\left(1-\psi_{X}^{2}\right) \\
& \times U^{L, T}\left(\kappa, \tau, \rho\left(\psi_{X}\right)\right),
\end{aligned}
$$

with

$$
\psi_{X}^{\max }(\kappa, \lambda)=\min \left\{1,\left(\frac{\kappa \sqrt{\frac{1}{\tau}+\rho_{\text {thresh }}^{2}-\lambda \rho_{\text {thresh }}-1}}{\xi_{F}}\right)^{1 / 2}\right\}
$$

and

$$
\frac{\partial \rho}{\partial \psi_{X}}=-\sqrt{2 \xi_{F}} \frac{\kappa\left(1+\xi_{F} \psi_{X}^{2}\right)-\lambda \psi_{X} \sqrt{2 \xi_{F}\left(1+\frac{1}{2} \xi_{F} \psi_{X}^{2}\right)}}{\tau \sqrt{1+\frac{1}{2} \xi_{F} \psi_{X}^{2}}} .
$$

In Eqs. (36) and (37) the function

$$
\begin{aligned}
\mathcal{D}(\kappa, \tau, \rho)= & \frac{1}{\epsilon_{F}-\epsilon_{0}(\rho)} \int_{\epsilon_{0}(\rho)}^{\epsilon_{F}} d \overline{\boldsymbol{\epsilon}} \int_{0}^{2 \pi} \frac{d \Phi}{2 \pi}(\boldsymbol{\eta} \times \hat{\boldsymbol{\kappa}})^{2} \\
= & \frac{\tau}{\kappa^{2}}\left\{\frac{1}{3}\left[\epsilon_{F}^{2}+\epsilon_{F} \epsilon_{0}(\rho)+\epsilon_{0}(\rho)^{2}\right]\right. \\
& \left.+\lambda\left[\epsilon_{F}+\epsilon_{0}(\rho)\right]+\lambda^{2}\right\}-(1+\tau)+(\rho-1) \\
& \times \frac{\tau}{\kappa^{2}}\left\{\lambda\left[\epsilon_{F}+\epsilon_{0}(\rho)\right]-\tau(\rho+1)\right\} \\
= & \xi_{F}\left(1-\psi_{X}^{2}\left[1+\xi_{F} \psi_{X}^{2}-\frac{\lambda}{\kappa} \psi_{X} \sqrt{\xi_{F}\left(2+\xi_{F} \psi_{X}^{2}\right)}\right.\right. \\
& \left.+\frac{\tau}{3 \kappa^{2}} \xi_{F}\left(1-\psi_{X}^{2}\right)\right]
\end{aligned}
$$

arises from the Fermi motion and goes to zero as $\xi_{F} \rightarrow 0$; being proportional to $\xi_{F} \cong \eta_{F}^{2} / 2 \ll 1$, this provides relatively moderate corrections to the rest of the contributions in Eqs. (36) and (37).

The value $\rho=1$ corresponds to QE kinematics: in this case the well-known expressions for the QE responses are recov- ered. The "total" observables are then obtained by adding the usual RFG QE response to the inelastic results:

$$
R_{\text {tot }}^{L, T}=R_{Q E}^{L, T}+R_{\text {inel }}^{L, T} .
$$

In the deep inelastic regime it is customary to deal with nuclear structure functions $W_{1,2}^{A}$ and/or $F_{1,2}^{A}$. These can be expressed in terms of the longitudinal and transverse response functions through the following relations:

$$
W_{1}^{A}=\frac{1}{2} R^{T},
$$

$$
W_{2}^{A}=\left(\frac{\tau}{\kappa^{2}}\right)^{2} R^{L}+\frac{1}{2} \frac{\tau}{\kappa^{2}} R^{T}
$$

and

$$
\begin{gathered}
F_{1}^{A}=m_{N} W_{1}^{A}, \\
F_{2}^{A}=2 m_{N} \lambda W_{2}^{A} .
\end{gathered}
$$

\section{B. Effects of binding energy}

In the study of superscaling for inclusive QE electron scattering from nuclei, an appropriate scaling variable $\psi^{\prime}$ was introduced by including a small energy shift to have the $\mathrm{QE}$ peak occur at the place where the scaling variable is zero. A detailed study of the sensitivity of the scaling function to variations of the Fermi momentum and energy shift was presented in Refs. $[16,18,20]$. Here we extend this analysis to the inelastic region. In principle, the introduction of an energy shift $\omega_{\text {shift }}$ in the formalism is straightforward and the calculation of the inelastic responses proceeds as in the $\omega_{\text {shift }}=0$ case. However, as will be made clear in the following, some complications arise. First, due to the general form assumed for the single-nucleon inelastic hadronic tensor, a certain asymmetry appears between the energy shift effects in the longitudinal and transverse responses. These shift effects are larger in the longitudinal response. Notice that this asymmetry already enters at the level of the QE nuclear responses. Second, there exists an ambiguity in the definition of the variable which should be used as the Bjorken $x$-scaling variable corresponding to the moving nucleon.

The effects of the inclusion of an energy shift on the inelastic nuclear hadronic tensor have been studied in the literature, with particular emphasis on the structure function $F_{2}$ and the European Muon Collaboration (EMC) effect at large values of $x$, in the context of so called "binding models" (see, for example, Refs. [30,31] and the general reviews [12,13]). The approach we follow here is the self-consistent generalization of previous works on the RFG. It is formally similar to the binding model approach, where in general the on-shell energy of the initial nucleon is modified by subtracting a constant term which effectively accounts for the nucleon separation energy and for the possibility that the residual nuclear system is left in an (highly) excited state. However, since the existing models either focus only on EMC ratios and/or use more realistic, although generally nonrelativistic wave functions, a precise quantitative comparison with those models is not possible. As we will discuss in the Results 
section, our calculations still miss some ingredients, coming from meson-exchange currents, and this makes a detailed quantitative comparison with experimental data premature; it is clear that, when this comparison will be made in the future, a more in-depth study of binding effects will also be needed.

In the RFG the energy shift is usually introduced by modifying the argument of the $\delta$ function appearing in the general expression of the inelastic hadronic tensor in Eq. (12), according to $\omega+\bar{E}_{\mathbf{h}}-E_{X} \rightarrow \omega^{\prime}+\bar{E}_{\mathbf{h}}-E_{X}$, where $\omega^{\prime}=\omega$ $-\omega_{\text {shift }}$. Then, introducing the invariant mass $W_{X}^{\prime 2} \equiv E_{X}^{2}-\mathbf{p}_{X}^{2}$ $=\left(\omega^{\prime}+\bar{E}_{\mathbf{h}}\right)^{2}-\mathbf{p}_{X}^{2}$, we can write the inelastic hadronic tensor in the form

$$
\begin{aligned}
W_{\text {inel }}^{\mu \nu}(\kappa, \lambda)= & \frac{3 \mathcal{N}}{4 \pi \eta_{F}^{3}} \int d \mu_{X}^{\prime} \int d \boldsymbol{\eta} \frac{\mu_{X}^{\prime}}{\bar{\epsilon} \epsilon_{X}} w_{i n e l}^{\mu \nu}\left(\boldsymbol{\eta}, \mu_{X}^{\prime} ; \kappa, \lambda\right) \\
& \times \delta\left(2 \lambda^{\prime}+\bar{\epsilon}-\epsilon_{X}\right) \theta\left(\eta_{F}-\eta\right),
\end{aligned}
$$

where $\mu_{X}^{\prime}=W_{X}^{\prime} / m_{N}$ and $\lambda^{\prime}=\omega^{\prime} /\left(2 m_{N}\right)$. As in the unshifted analysis, the $\delta$ function can be used to perform the polar angular integration, leading to the result

$$
\begin{aligned}
W_{\text {inel }}^{\mu \nu}(\kappa, \lambda)= & \frac{3 \mathcal{N} \tau^{\prime}}{2 \eta_{F}^{3} \kappa} \int_{\rho_{1}^{\prime}\left(\kappa, \lambda^{\prime}\right)}^{\rho_{2}^{\prime}\left(\kappa, \lambda^{\prime}\right)} d \rho^{\prime} \int_{0}^{2 \pi} \\
& \times \frac{d \Phi}{2 \pi} \int_{\epsilon_{0}^{\prime}\left(\rho^{\prime}\right)}^{\epsilon_{F}} d \bar{\epsilon} w_{i n e l}^{\mu \nu}\left(\bar{\epsilon}, \rho^{\prime} ; \kappa, \lambda\right),
\end{aligned}
$$

where the variable $\rho^{\prime}$ is defined as

$$
\rho^{\prime} \equiv \frac{2 H \cdot Q^{\prime}}{\left|Q^{\prime 2}\right|}=\left[1+\frac{1}{4 \tau^{\prime}}\left(\mu_{X}^{\prime 2}-1\right)\right]
$$

and $\tau^{\prime} \equiv \kappa^{2}-\lambda^{\prime 2}$.

The inclusion of the energy shift modifies the integration limits over $\bar{\epsilon}$ in the following way:

$$
\bar{\epsilon} \geqslant \epsilon_{0}^{\prime}\left(\rho^{\prime}\right) \equiv \kappa \sqrt{\frac{1}{\tau^{\prime}}+\rho^{\prime 2}}-\lambda^{\prime} \rho^{\prime} .
$$

Correspondingly, the region for the integration over $\rho^{\prime}$ is given by

$$
\begin{aligned}
{\left[\rho_{1}^{\prime}\left(\kappa, \lambda^{\prime}\right), \rho_{2}^{\prime}\left(\kappa, \lambda^{\prime}\right)\right]=} & {\left[\operatorname { m a x } \left\{\frac{\lambda^{\prime} \epsilon_{F}-\kappa \eta_{F}}{\tau^{\prime}},\right.\right.} \\
& \left.\left.1+\frac{\mu_{\pi}}{4 \tau^{\prime}}\left(2+\mu_{\pi}\right)\right\}, \frac{\lambda^{\prime} \epsilon_{F}+\kappa \eta_{F}}{\tau^{\prime}}\right] .
\end{aligned}
$$

The definition of the inelastic scaling variable becomes now

$$
\psi_{X}^{\prime 2} \equiv \frac{\epsilon_{0}^{\prime}\left(\rho^{\prime}\right)-1}{\epsilon_{F}-1}
$$

and the inelastic longitudinal and transverse response functions, calculated as $R_{\text {inel }}^{L}=W_{\text {inel }}^{00}$ and $R_{\text {inel }}^{T}=W_{\text {inel }}^{11}+W_{\text {inel }}^{22}$, have the following general forms:

$$
R_{\text {inel }}^{L, T}(\kappa, \tau)=\frac{3 \mathcal{N} \tau^{\prime}}{2 \eta_{F}^{3} \kappa} \xi_{F} \int_{\rho_{1}^{\prime}\left(\kappa, \lambda^{\prime}\right)}^{\rho_{2}^{\prime}\left(\kappa, \lambda^{\prime}\right)} d \rho^{\prime}\left(1-\psi_{X}^{\prime 2}\right) U^{L, T}\left(\kappa, \tau, \rho^{\prime}\right)
$$

In order to evaluate the longitudinal and transverse nuclear functions $U^{L, T}\left(\kappa, \tau, \rho^{\prime}\right)$ one needs to assume a specific form for the inelastic single-nucleon tensor $w_{i n e l}^{\mu \nu}\left(\bar{\epsilon}, \rho^{\prime} ; \kappa, \lambda\right)$. It is important to remark that there exists some ambiguity in the choice made here: for instance, several alternatives involving different expressions containing the four-momenta $Q^{\mu}$ and/or $Q^{\prime \mu}$ are possible, and these can lead to different results. For example, in Ref. [30], the modified four momentum transfer $Q^{\prime \mu}$ is used, although then a prescription must be used in order to recover the gauge invariance of the nuclear hadronic tensor, which is lost by making this choice. In Appendix B we present the specific expressions of the inelastic and $\mathrm{QE}$ responses obtained for a given selection of the singlenucleon tensors accounting for the energy shift. Apart from the specific form of the tensor $w^{\mu \nu}$, the choice of the arguments of the single-nucleon inelastic structure functions, $w_{1}, w_{2}$, also presents some ambiguities. In fact, the available parametrizations for $w_{1}, w_{2}$ that we employ in our calculations are given for free, on-shell, nucleons, while the inclusion of the energy shift effectively introduces some "offshellness" of the initial nucleon, by altering the energy balance at the vertex where it couples to the exchanged virtual photon. In this case the bound-nucleon Bjorken variable is not uniquely determined by the final-state invariant mass and, since no theoretically derived prescriptions exist, one has to make some assumptions. As shown in Appendix B, the inelasticity parameter selected in this work, $\tilde{\rho}$, corresponds to the one given as $\left(2 m_{N} \widetilde{\omega}\right) /\left|Q^{2}\right|=\rho^{\prime}\left(\tau^{\prime} / \tau\right)$, where $\widetilde{\omega}$ is the energy transferred to the nucleon in the system in which the nucleon is at rest. This means that in our numerical calculations, for a given set of values of $\omega, Q^{2}$, and $\rho^{\prime}$ we employ free-nucleon structure functions taken at fourmomentum $Q^{2}$ and Bjorken variable $1 / \widetilde{\rho}$.

\section{Extended relativistic fermi gas}

As discussed in previous works $[8,16,18,20]$, for a fixed value of the invariant mass $\mu_{X}$, the RFG yields a scaling function

$$
f\left(\psi_{X}^{\prime}\right)=f_{L}\left(\psi_{X}^{\prime}\right)=f_{T}\left(\psi_{X}^{\prime}\right)=\frac{3}{4}\left(1-\psi_{X}^{\prime 2}\right) \theta\left(1-\psi_{X}^{\prime 2}\right)
$$

which, as a function of the appropriate scaling variable $\psi_{X}^{\prime}$, is the same for all values of $\mu_{X}{ }^{1}$

In Ref. [18] the behavior of the longitudinal scaling function was studied for the existing world data in the QE region. This study showed that to a good approximation $f_{L}\left(\psi^{\prime}\right)$ superscales, that is, it does not show any significant dependence on the momentum transfer $\kappa$ (scaling of the first kind) and is approximately the same for all nuclear species (scal-

\footnotetext{
${ }^{\mathrm{I}}$ The function in Eq. (54) differs from the one used in previous work [20] by a multiplicative function $2 \xi_{F} / \eta_{F}^{2}\left[1+\frac{1}{2} \xi_{F}\left(1+\psi_{X}^{\prime 2}\right)\right]$. We have checked that this is numerically unimportant for all of the kinematical conditions considered here.
} 


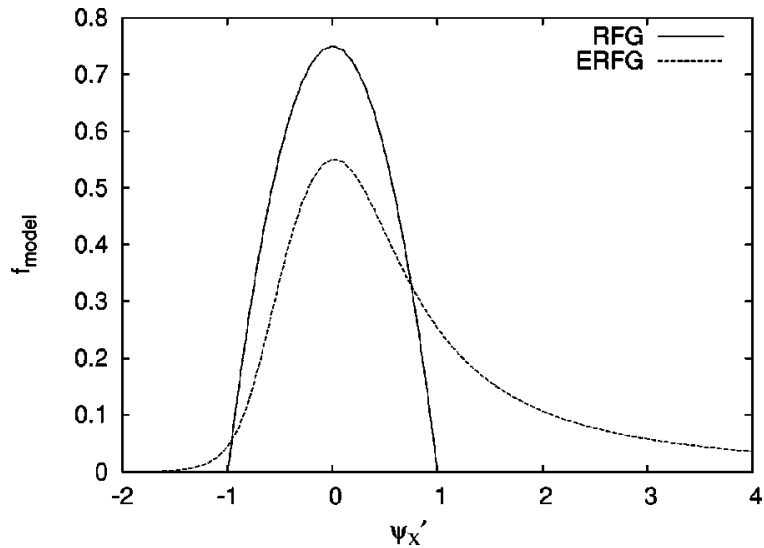

FIG. 1. Scaling function $f_{\text {model }}\left(\psi_{X}^{\prime}\right)$ of Eq. (58) for the RFG and ERFG models.

ing of the second kind). An expression for a phenomenological longitudinal scaling function, $f_{\text {univ }}\left(\psi^{\prime}\right)$, was obtained by fitting the data [21]. Based on these results, we now make the following hypothesis: we assume that this $f_{\text {univ }}\left(\psi^{\prime}\right)$, derived from the data, provides a good description of $f\left(\psi_{X}^{\prime}\right)=f_{L}\left(\psi_{X}^{\prime}\right)$ $=f_{T}\left(\psi_{X}^{\prime}\right)$, ("scaling of the zeroth kind") as it implicitly contains the initial-state physics, and thus we make, for any $\mu_{X}$, the following substitution:

$$
\frac{3}{4}\left(1-\psi_{X}^{\prime 2}\right) \theta\left(1-\psi_{X}^{\prime 2}\right) \rightarrow f_{E R F G}\left(\psi_{X}^{\prime}\right)=f_{\text {univ }}\left(\psi_{X}^{\prime}\right) .
$$

To be more specific, we calculate the response functions as

$$
\begin{gathered}
R_{Q E}^{L, T}=\frac{\mathcal{N}}{\eta_{F}^{3} \kappa m_{N}} \xi_{F} f_{\text {model }}\left(\psi^{\prime}\right) U_{Q E}^{L, T}, \\
R_{\text {inel }}^{L, T}(\kappa, \tau)=\frac{\mathcal{N}}{\eta_{F}^{3} \kappa} \xi_{F} \int_{\mu_{\text {thresh }}}^{1+2 \lambda-\epsilon_{S}} d \mu_{X} \mu_{X} f_{\text {model }}\left(\psi_{X}^{\prime}\right) U^{L, T},
\end{gathered}
$$

where $\epsilon_{S}=E_{S} / m_{N}$ is the dimensionless separation energy and

$$
f_{\text {model }}\left(\psi_{X}^{\prime}\right)=\left\{\begin{array}{l}
\frac{3}{4}\left(1-\psi_{X}^{\prime 2}\right) \theta\left(1-\psi_{X}^{\prime 2}\right) \quad \text { model }=\mathrm{RFG} \\
f_{\text {univ }}\left(\psi_{X}^{\prime}\right) \quad \text { model }=\mathrm{ERFG} .
\end{array}\right.
$$

The functions $f_{R F G}$ and $f_{E R F G}$ are shown in Fig. 1 as functions of $\psi_{X}^{\prime}$, while the functions $U_{Q E}^{L, T}$ and $U^{L, T}$ in Eqs. (56) and (57) are given in Appendix B.

\section{RESULTS}

In this section we present our results for cross sections and response and structure functions. In computing the inelastic hadronic tensor of Eq. (47), we employ phenomenological fits of the single-nucleon inelastic structure functions. The latter are measured in DIS experiments and a variety of parametrizations for $w_{1}$ and $w_{2}$ can be found in the literature [15,32-36], including some variations arising from the different assumptions made for how to extract the neutron structure functions from deuteron data. Unless stated otherwise, in the following we adopt the Bodek et al. fit of Refs. $[15,32,33]$, which describes both the deep inelastic and reso-

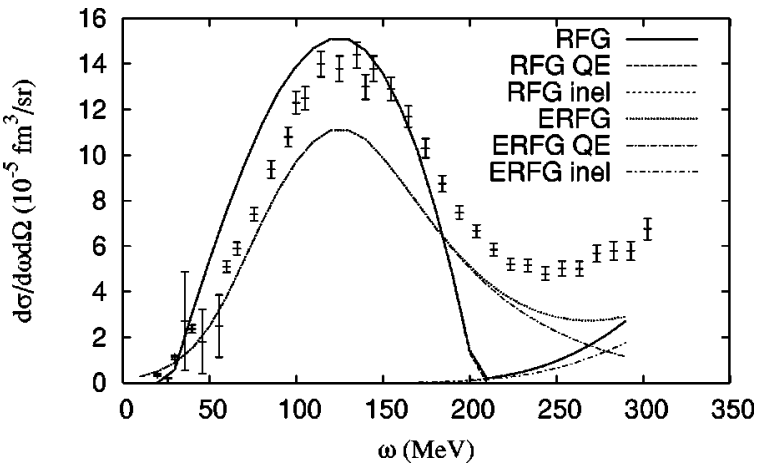

FIG. 2. Inclusive cross section for electron scattering from carbon at $E_{i n c}=500 \mathrm{MeV}$ and $\theta_{e}=60^{\circ}$ vs the energy transfer. The calculation includes an energy shift $\omega_{\text {shift }}=20 \mathrm{MeV}$ and the separate QE and inelastic contributions to the cross section are shown. Data are from Ref. [41].

nance regions. For the QE contributions, we employ the form factor parametrization of Ref. [37]. The sensitivity of the results to the different parametrization choices will be discussed later.

Additionally, for the Fermi momentum and the energy shift we will employ the values obtained in Ref. [20], namely, $k_{F}=220 \mathrm{MeV} / c, \omega_{\text {shift }}=20 \mathrm{MeV}$ for carbon, $k_{F}$ $=236 \mathrm{MeV} / c, \quad \omega_{\text {shift }}=18 \mathrm{MeV}$ for aluminum, $k_{F}$ $=241 \mathrm{MeV} / c, \omega_{\text {shift }}=23 \mathrm{MeV}$ for iron, and $k_{F}=245 \mathrm{MeV} / c$, $\omega_{\text {shift }}=25 \mathrm{MeV}$ for gold.

\section{A. Cross sections}

In this section we present our results for the cross sections in the RFG and ERFG models and compare them with the available experimental data [38-41].
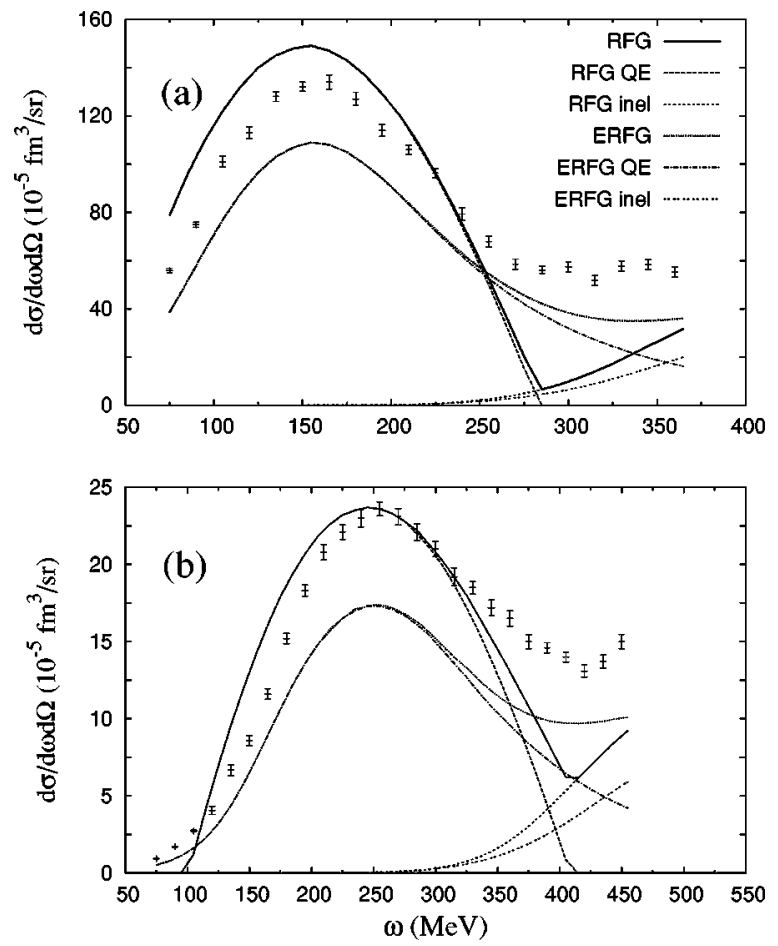

FIG. 3. As for Fig. 2, but at $E_{i n c}=2.020 \mathrm{GeV}$ and scattering angle $\theta_{e}=15^{\circ}$ (a) and $\theta_{e}=20^{\circ}$ (b). Data are from Ref. [39]. 

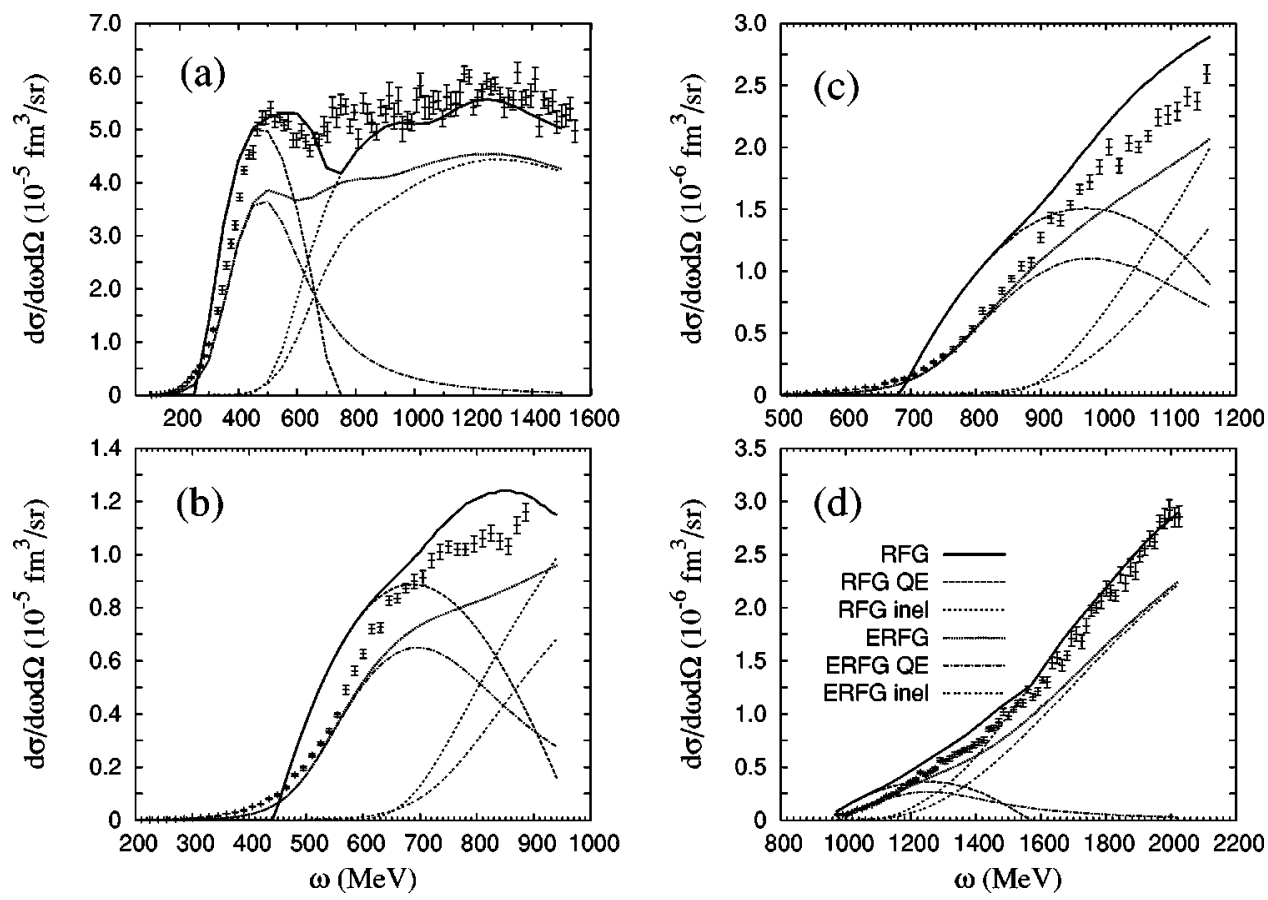

FIG. 4. As for Fig. 2, but at $E_{e}=3.595 \mathrm{GeV}$ and scattering angle $\theta_{e}=16^{\circ}$ (a), $20^{\circ}$ (b), $25^{\circ}$ (c), and $30^{\circ}(\mathrm{d})$. Data are from Ref. [39].

In Fig. 2 we show the inclusive cross section for a ${ }^{12} \mathrm{C}$ target at $E_{e}=500 \mathrm{MeV}$ and $\theta_{e}=60^{\circ}$. We separate the $\mathrm{QE}$ from the inelastic contribution. We notice that the shifted RFG model (solid line) yields roughly the right position and height of the QE peak, but fails to reproduce the tails of the peak, giving in particular an unobserved dip at $\omega$ $\simeq 200 \mathrm{MeV}$. On the other hand the ERFG model (dotted line), while reproducing the data in the tails better, significantly underestimates the cross section at the peak. This is related to the fact that, as shown in Fig. 1, the peak of the ERFG universal function $f_{E R F G}$ is lower than the corresponding RFG value. Due to the larger extension of $f_{E R F G}$ over $\psi_{X}^{\prime}$ the normalization of the two functions is the same, namely, $\int f_{R F G} d \psi_{X}^{\prime}=\int f_{E R F G} d \psi_{X}^{\prime}=1$. One might then naively expect the integral in Eq. (57) which yields the inelastic response functions to be the same in the two models. However, a closer inspection shows that this is not the case because the integration limits and/or the weighting provided by $U^{L, T}$ are such that the ERFG integral does not "saturate" as does the RFG one.

Figures 3-5 correspond to different kinematical conditions, namely, $E_{e}=2.020$ and $3.595 \mathrm{GeV}$ (SLAC) and $E_{e}$ $=4.045 \mathrm{GeV}(\mathrm{JLab})$ and various scattering angles. Concerning Figs. 3(a) and 3(b), a similar trend persists, with the
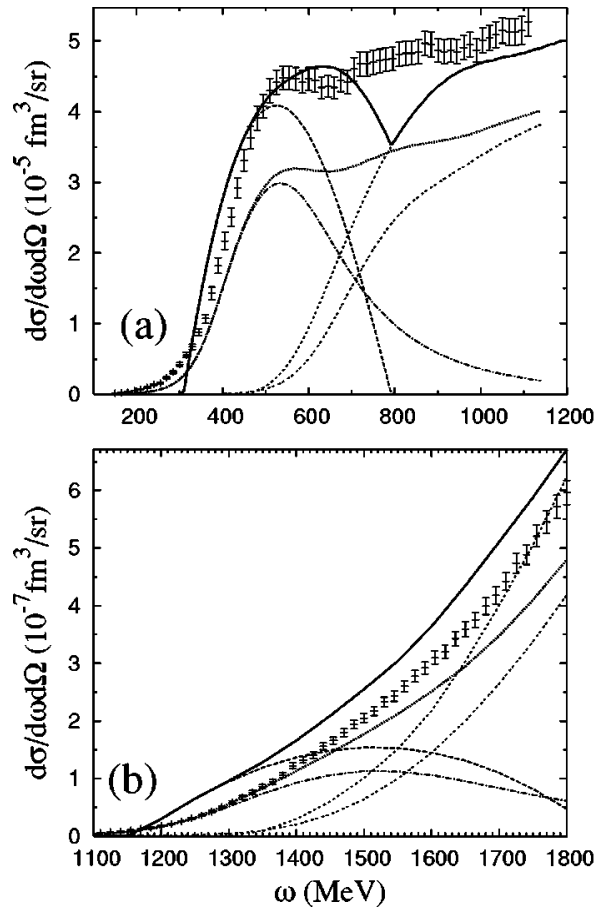
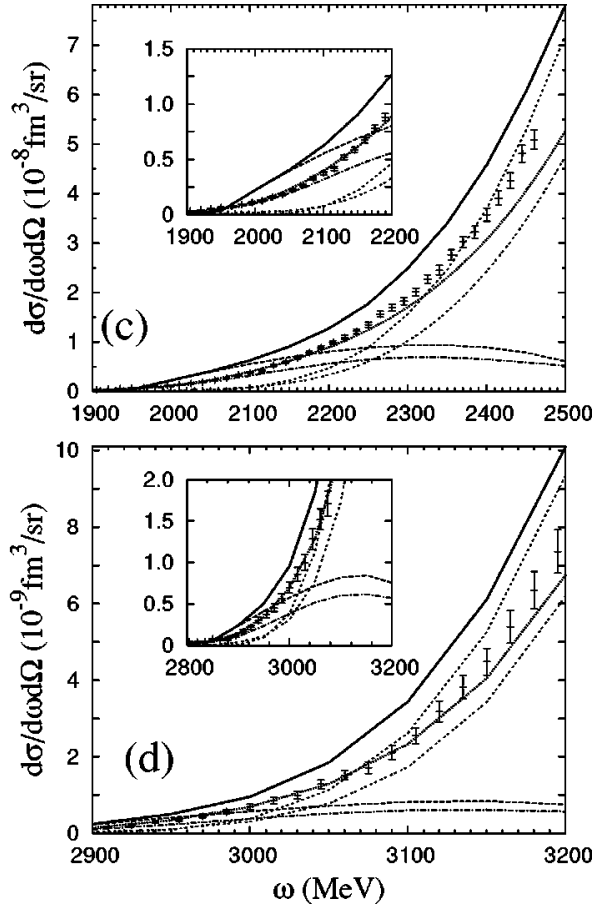

$035502-8$
FIG. 5. As for Fig. 2, but at $E_{\text {inc }}=4.045 \mathrm{GeV}$ and scattering angle $\theta_{e}=15^{\circ}$ (a), $30^{\circ}$ (b), $45^{\circ}$ (c), and $74^{\circ}$ (d). Data are from Ref. [38]. 

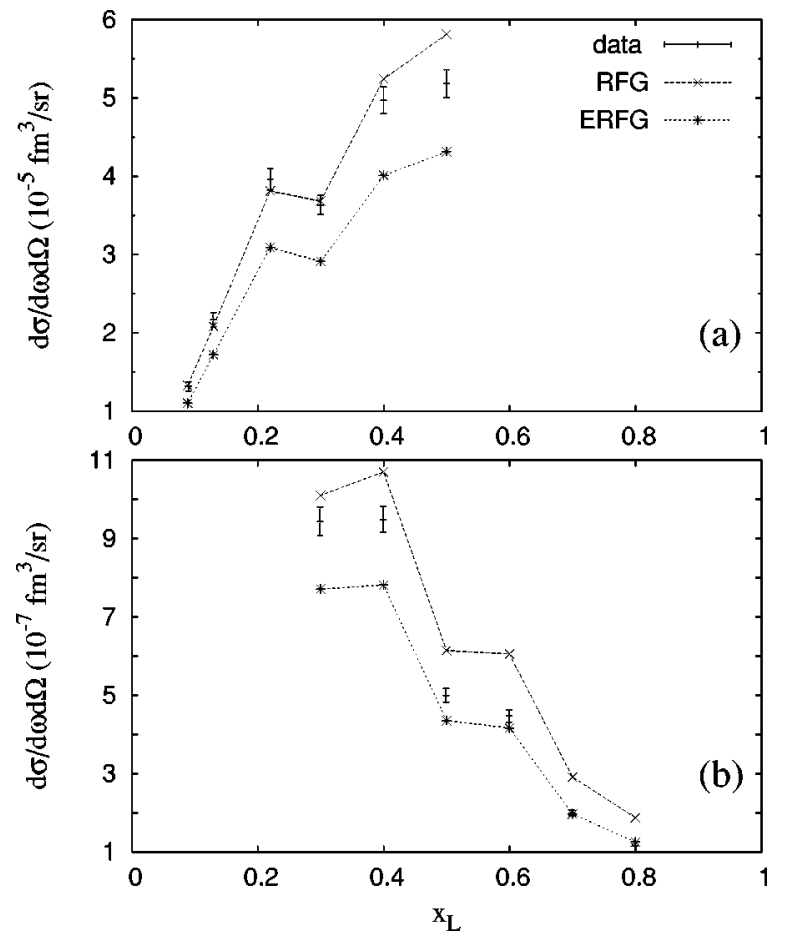

FIG. 6. Differential cross section $d \sigma / d \omega d \Omega_{e}$ for electron scattering on iron, shown as a function of $x_{L}$ at fixed $\left|Q^{2}\right|$. Panel (a): $\left|Q^{2}\right|=2(\mathrm{GeV} / c)^{2}$, the experimental points, from right to left, are taken at $\left[E_{e}(\mathrm{GeV}), \theta_{e}(\mathrm{deg})\right]=(8,11.8),(8,12.4),(8,13.6),(9.7$, $11.8),(12,11.8),(15,11.8)$. Panel (b): $\left|Q^{2}\right|=10(\mathrm{GeV} / c)^{2}$, the experimental points, from right to left, are taken at $\left[E_{e}(\mathrm{GeV}), \theta_{e}(\mathrm{deg})\right]=(15,16.0),(15,17.1),(17,15.0),(17,16.9),(21$, 14.1),(24,14.1). Data are from Ref. [40].

ERFG model significantly underestimating the data in the region of the QE peak, whereas the RFG is closer to the data (particularly for $\theta_{e}=20^{\circ}$ ), although it leaves no room for other contributions to be added. Note also that for this scattering angle the inelastic channel starts to be sizable.

Examining Figs. 4 we remark that the QE peak, which is more clearly separated from the inelastic region in Fig. 4(a), is again well reproduced in the low- $\omega$ tail by the ERFG, while its maximum agrees better with the RFG. On the other hand the inelastic cross section is in all cases underestimated by the ERFG, while the RFG alone would roughly account for what is observed.

Similar comments apply to Fig. 5(a), corresponding to higher energy and low scattering angle. For higher angles [Figs. 5(b)-5(d)] the data lie roughly in between the predictions of the ERFG (smaller) and RFG (larger) models, the former again reproducing the low- $\omega$ behavior better. As a general result we observe that as the scattering angle increases the range of validity of the ERFG also increases.

Finally, in Figs. 6 and 7 we consider slightly different kinematical conditions, corresponding to fixed values of $\left|Q^{2}\right|$ in the range $2-10(\mathrm{GeV} / c)^{2}$, and various electron energies (in the range $8-25 \mathrm{GeV}$ ) and angles $\left(12^{\circ}-22^{\circ}\right)$. Theoretical results for ${ }^{56} \mathrm{Fe}$ are shown as functions of the "laboratory" Bjorken variable $x_{L}$. The data corresponding to a fixed $Q^{2}$ are taken at different values of $E_{e}$ and $\theta_{e}$. For $\left|Q^{2}\right|=2$ and $10(\mathrm{GeV} / c)^{2}$ (Fig. 6) the various data fit reasonably well on

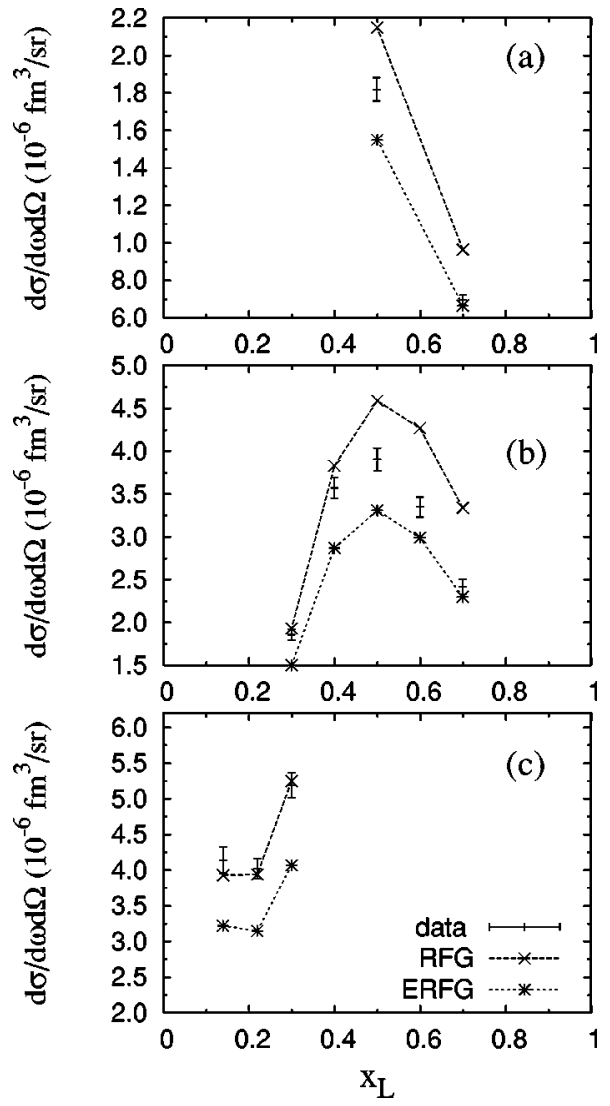

FIG. 7. As for Fig. 6, but at $\left|Q^{2}\right|=5(\mathrm{GeV} / c)^{2}$. The two data in the upper panel, from right to left, correspond to $E_{e}=8 \mathrm{GeV}$ and $\theta_{e}=22^{\circ}$ and to $E_{e}=9.7 \mathrm{GeV}$ and $\theta_{e}=19.7^{\circ}$; the data in the middle panel have fixed $E_{e}=12 \mathrm{GeV}$ and, from right to left, $\theta_{e}$ $=12.8,13.3,14.2,15.8,20.6^{\circ}$; the data in the lower panel, from right to left, are taken at $\left(E_{e}=15 \mathrm{GeV}, \theta_{e}=13.2^{\circ}\right),\left(E_{e}=17 \mathrm{GeV}, \theta_{e}\right.$ $\left.=13.5^{\circ}\right),\left(E_{e}=24.5 \mathrm{GeV} \theta_{e}=11.1^{\circ}\right)$. Data are from Ref. [40].

one plot, whereas for $\left|Q^{2}\right|=5(\mathrm{GeV} / c)^{2}$ (Fig. 7), for clarity we have separated the data into three sets as indicated in the figure caption. We notice that at large $x_{L}(\geqslant 0.6)$ the data are closer to the ERFG predictions, at low $x_{L}(0.1-0.3)$ they are closer to the RFG calculation and for $0.3 \leqslant x_{L} \leqslant 0.6$ they lie in between the two models. This general trend seems to be respected for all values of $Q^{2}$ (at least where data are available).

We have also analyzed the effect introduced by different electromagnetic form factor parametrizations ([42-44]) and verified that it can produce a $\pm 3 \%$ uncertainty at the $\mathrm{QE}$ peak, but does not change the general agreement/ disagreement of the models with the data. Moreover, it should be remarked that, at the energies considered in this section, the contribution from the resonance region to the inelastic part of the cross section is quite important and thus a comparison with results obtained by using purely DIS parametrizations [34,36] of the single-nucleon structure functions is not appropriate. At the highest $\left|Q^{2}\right|$ values considered here [Figs. 6(b) and 7], the use of different parametrizations $[34,36]$ does not produce significant variations in the results.

An important comment, already anticipated in the introduction, is in order. The RFG and ERFG models considered 

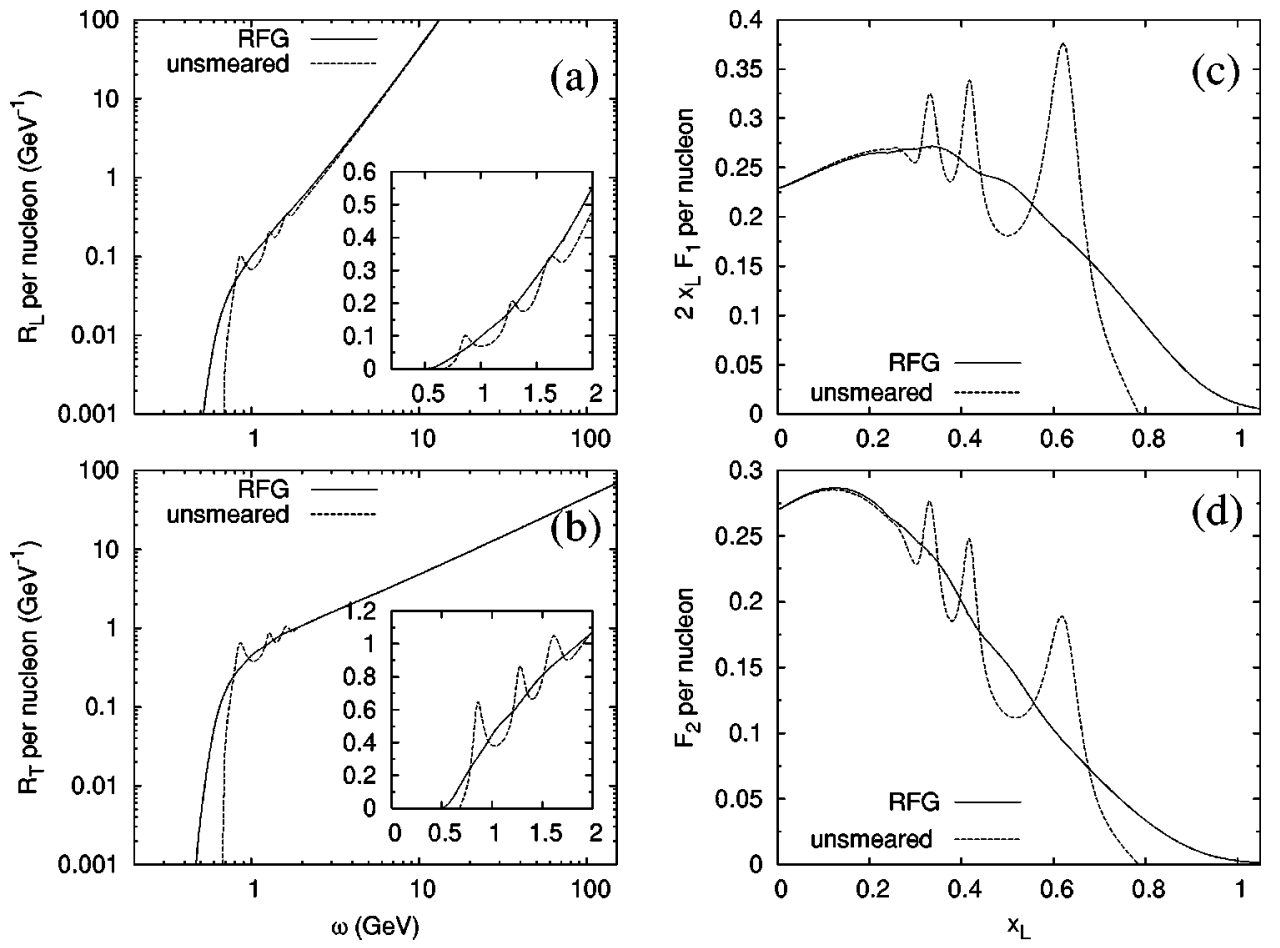

FIG. 8. Panels (a) and (b): inelastic and unsmeared response functions $R_{\text {inel }}^{L, T}$ per nucleon as functions of the energy transfer $\omega$ for ${ }^{56} \mathrm{Fe}$, calculated within the $\mathrm{RFG}$, with $k_{F}=241 \mathrm{MeV} / c$ and $\omega_{\text {shift }}=23 \mathrm{MeV}$. Panels (c) and (d): inelastic and unsmeared structure functions $2 x_{L} F_{1}^{A}$ and $F_{2}^{A}$ per nucleon as functions of $x_{L}=\tau / \lambda$. in this study include the $1 p-1 h$ one-body contributions both for elastic scattering from a nucleon in the nucleus and for representations of the single-nucleon inelastic spectrum, thereby incorporating effects from meson production, excitation of baryon resonances (notably the $\Delta$ ) and, at high excitation energies, DIS. However, this is not the entire story: in this region and beyond effects arising from reaction mechanisms not included here, namely, those coming from correlations and both $1 p$ - $1 h$ and $2 p$ - $2 h$ meson-exchange currents (MEC) are also important [3-8,20,22]. In particular, in a recent study [22] effects from $2 p$ - $2 h$ meson-exchange currents were explored for high-energy conditions where relativistic modeling is important. The resulting cross sections are significant in the region above the QE peak and therefore tend to bring the total (the present ERFG contributions plus these additional MEC contributions) into better agreement with the data. While this is encouraging, it is still not the full story, since the $2 p$ - $2 h$ MEC contributions have corresponding correlation contributions, as required by gauge invariance (and as was studied in detail in our previous work on $1 p-1 h$ MEC plus correlation effects [7]). The $2 p-2 h$ correlations have not yet been incorporated and thus detailed comparisons with data are somewhat premature.

In summary, the RFG model clearly overestimates the low- $\omega$ data, which are better reproduced by the ERFG model (dotted line), and the fact that the latter yields a cross section that is below the data is encouraging, since this leaves room for the above-mentioned effects to provide the balance.

\section{B. Response and structure functions}

In the RFG framework the only effect of the nuclear medium arises from the Fermi motion of the nucleons inside the nucleus. To quantify the impact of the Fermi smearing on the observables we have compared the inelastic RFG response functions with the corresponding "unsmeared" ones

$$
R_{\text {unsm. }}^{L, T} \equiv Z R_{\text {proton }}^{L, T}+N R_{\text {neutron }}^{L, T}
$$

where $R_{\text {proton(neutron) }}^{L, T}$ are the response functions for a free proton (neutron) at rest in the laboratory frame. ${ }^{2}$

A similar comparison has been done for the nuclear structure functions $W_{1,2}^{A}$ and/or $F_{1,2}^{A}$ of Eqs. (43)-(46).

In presenting the results we choose the following kinematical conditions: we select a relatively low (but typical) four-momentum transfer $\tau=0.284$ [corresponding to $\left|Q^{2}\right|$ $\left.=1(\mathrm{GeV} / c)^{2}\right]$, in order to illustrate the differences between smeared and unsmeared quantities better. The calculations are performed for the case of ${ }^{56} \mathrm{Fe}$ with $k_{F}=241 \mathrm{MeV} / c$ and they include the energy shift discussed in Sec. III B, with $\omega_{\text {shift }}=23 \mathrm{MeV}$.

In Fig. 8(a) and 8(b) the inelastic response functions $R_{\text {inel }}^{L, T}$ per nucleon are plotted as functions of the energy transfer $\omega$, while in Figs. 8(c) and 8(d) the structure functions $2 x_{L} F_{1}$ and $F_{2}$ are shown as functions of $x_{L}$. It is seen that in the resonance region [small panels in Fig. 8(a) and 8(b)] the Fermi smearing effects are rather large and completely smooth out

\footnotetext{
${ }^{2}$ The Bodek et al. fit we employ to describe the single-nucleon structure functions was obtained from data on cross sections assuming a constant ratio $\sigma_{L} / \sigma_{T}=0.18$. When the fit is used to evaluate the separate responses at relatively low $\left|Q^{2}\right|$, this may lead to some "spurious" effects, such as the bump observed in the unsmeared $R_{L}$ at $\omega=0.86 \mathrm{GeV}$, corresponding to the $\Delta$ resonance. This indicates that new and more precise fits of the nucleon structure functions in the resonance region are needed.
} 

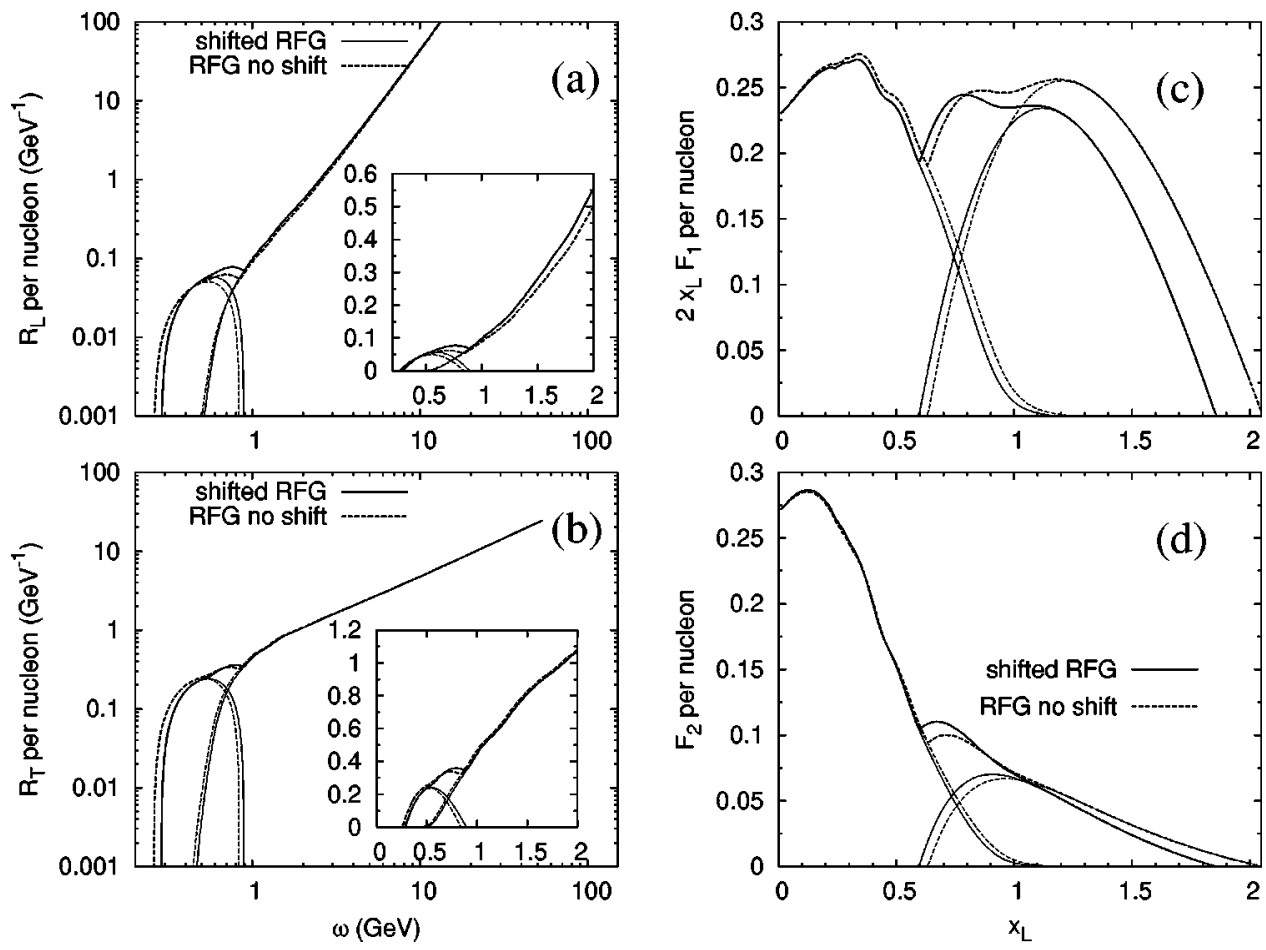

FIG. 9. Panels (a) and (b): response functions $R_{L}$ and $R_{T}$ per nucleon, respectively, as functions of $\omega$ for ${ }^{56} \mathrm{Fe}$. The separate contributions of the QE peak (visible at low $\omega$ ) and of the inelastic response functions are shown, together with the sum of the two contributions. Panels (c) and (d): structure functions $2 x_{L} F_{1}$ and, respectively, $F_{2}$ per nucleon vs $x_{L}$. In this case the contribution of the QE peak is the one on the right (large $x_{L}$ ) and, correspondingly, the inelastic contribution is the structure appearing at small $x_{L}$. the resonance structure of the single-nucleon responses, while in the DIS region (large $\omega$ or small $x_{L}$ ) almost no effect of the nuclear medium is observed.

To illustrate the kind of effects introduced by the energy shift accounted for following the procedure presented in Appendix B, we show in Figs. 9(a) and 9(b) the hadronic responses, $R^{L, T}$, as functions of $\omega$. The energy shift has been taken to be $\omega_{\text {shift }}=23 \mathrm{MeV}$. We present separately the QE and inelastic channel contributions as well as the global result. A similar analysis for the structure functions $2 x_{L} F_{1}^{A}$ and $F_{2}^{A}$, as given in Eqs. (45) and (46), is presented in Fig. 9(c) and $9(\mathrm{~d})$. Note that the effects introduced by the energy shift are observable in the QE peak and tend to disappear increasingly rapidly when moving to the inelastic region. In particular, it is interesting to remark that the longitudinal response seems to be more sensitive to inclusion of the energy shift. This is connected with the large terms entering in $\mathcal{D}_{L}^{\prime}$ (see Appendix B). In this case the energy shift effects remain evident even at very large $\omega$.

\section{Scaling functions}

In this section we investigate more closely the secondkind scaling behavior within the context of the inelastic RFG model. Since the second-kind scaling analysis involves comparisons of different nuclear species at the same kinematics and since a large "reach" in density (or equivalently in Fermi momentum) is advantageous, we add the case of gold to the discussions above.

In Fig. 10 we plot the inclusive cross sections on gold for the kinematical conditions $E_{i n c}=3.6 \mathrm{GeV}, \theta_{e}=16^{\circ}$, and compare them with available experimental data, taken at SLAC [39].

Since it has been found to be desirable to have separate information on longitudinal and transverse responses when discussing second-kind scaling, we proceed as in past work [20] where these data (for carbon, aluminum, iron, and gold) were used to obtain " $L$-subtracted" transverse response functions and then transverse superscaling functions. The $L$ subtraction was performed by assuming a universal longitudinal superscaling function $f_{L}^{\text {universal }}\left(\psi^{\prime}\right)=f_{E R F G}\left(\psi^{\prime}\right)$ (see Sec. III C) and reconstructing from it the longitudinal cross section:

$$
\Sigma_{L}=\frac{f_{L}^{\text {universal }}}{k_{F}} v_{l} G_{L} \sigma_{M} .
$$

$\Sigma_{L}$ was then subtracted from the total inclusive cross section in order to obtain

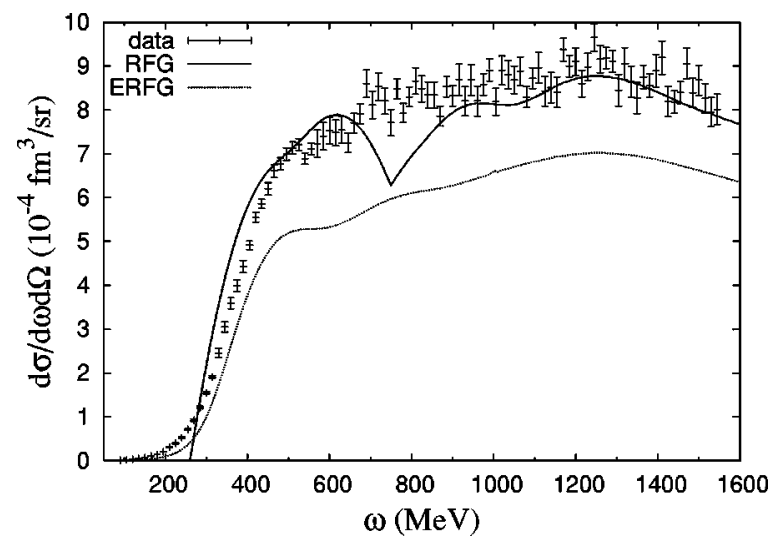

FIG. 10. Inclusive cross section for electron scattering from gold, at $E_{\text {inc }}=3.6 \mathrm{GeV}$ and $\theta_{e}=16^{\circ}$ vs the energy transfer. Data are from Ref. [39]. 


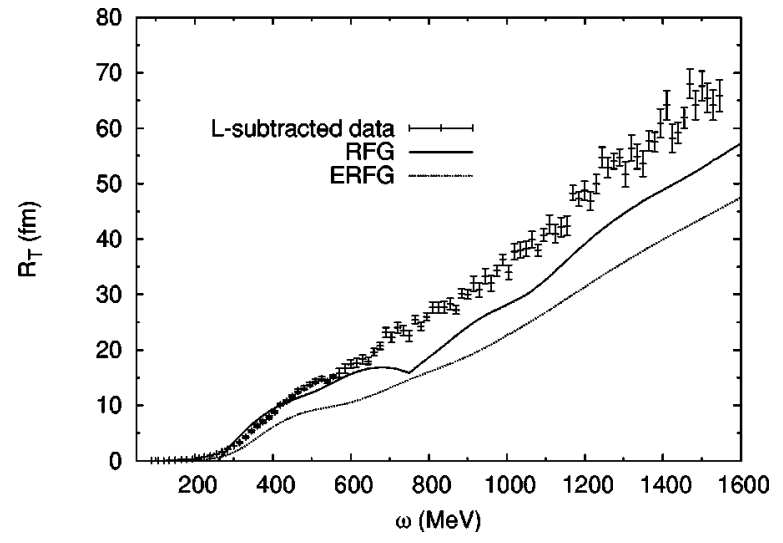

FIG. 11. Transverse response function for electron scattering from gold, at $E_{i n c}=3.6 \mathrm{GeV}$ and $\theta_{e}=16^{\circ}$ vs the energy transfer. The "data" are obtained from measured inclusive cross sections by means of the $L$-subtraction procedure described in the text.

$$
\Sigma_{T}=d \sigma-\Sigma_{L}
$$

and then

$$
\begin{gathered}
R^{T}=\frac{\Sigma_{T}}{\sigma_{M} v_{T}}, \\
f_{T}=\frac{k_{F} R^{T}}{G_{T}} .
\end{gathered}
$$

In the above equations, according to Ref. [20],

$$
\begin{aligned}
& G_{L}=\frac{\left(\kappa^{2} / \tau\right)\left[\tilde{G}_{E}^{2}+\widetilde{W}_{2} \Delta_{T}^{\prime}\right]}{2 \kappa\left[1+\xi_{F}\left(1+\psi^{\prime 2}\right) / 2\right]}, \\
& G_{T}=\frac{2 \tau \widetilde{G}_{M}^{2}+\widetilde{W}_{2} \Delta_{T}^{\prime}}{2 \kappa\left[1+\xi_{F}\left(1+\psi^{\prime 2}\right) / 2\right]}
\end{aligned}
$$

with $\Delta_{T}^{\prime}$ defined in Eq. (B11).
In Fig. 11 we show for the case of gold the " $L$-subtracted" (according to the procedure described above) data for $R^{T}$ and compare them with the theoretically calculated $R^{T}$, including both QE and inelastic contributions. Note that the transverse results obtained via this subtraction procedure display a shortfall at high inelasticity of "data" versus inelastic RFG modeling, which is not apparent in the total cross section shown in the previous figure. This can be due to the fact that in subtracting the longitudinal part, when elaborating the data, we may be using a longitudinal cross section that is too small, or, when assuming a certain parametrization for the single-nucleon ratio $w_{2} / w_{1}$ (related to $R$ ) to obtain the theoretical curves, we may be indirectly assuming a $\Sigma_{L}$ that is too large. Moreover, as discussed above, there is still a $2 p-2 h$ MEC plus correlation contribution to be taken into account (note that the $2 p-2 h$ MEC contribution is predominantly transverse and so this result is not unexpected).

Similar results are obtained for the superscaling functions $f\left(\psi^{\prime}\right)$ and $f_{T}\left(\psi^{\prime}\right)$. In Fig. 12 the total scaling function $f$ is shown as a function of the QE variable $\psi^{\prime}$ for the four nuclear species under discussion, within the RFG (left panel) and ERFG (right panel) models, at the same kinematics of Figs. 10 and 11; experimental data are obtained from the measured inclusive cross sections divided by

$$
\sigma_{M}\left(v_{L} G_{L}+v_{T} G_{T}\right)
$$

and the curves are obtained by dividing the theoretical inclusive cross section by the same quantity as in Eq. (66).

Finally, Fig. 13 (corresponding to Fig. 5 of Ref. [20]) shows the transverse superscaling function $f_{T}\left(\psi^{\prime}\right)$, at the same kinematics, again in the RFG and ERFG models. The "data" are obtained from the experimental inclusive cross sections according to Eqs. (60)-(63), while the curves are obtained by dividing the theoretical $R^{T}$ of Eqs. (42), (B8), and (53) by $G_{T}$. Again we observe that, the discrepancy between data and "theory" is larger for the transverse case than for the total scaling functions at this scattering angle $(\theta$ $\left.=16^{0}\right)$. This indicates that extra contributions should be

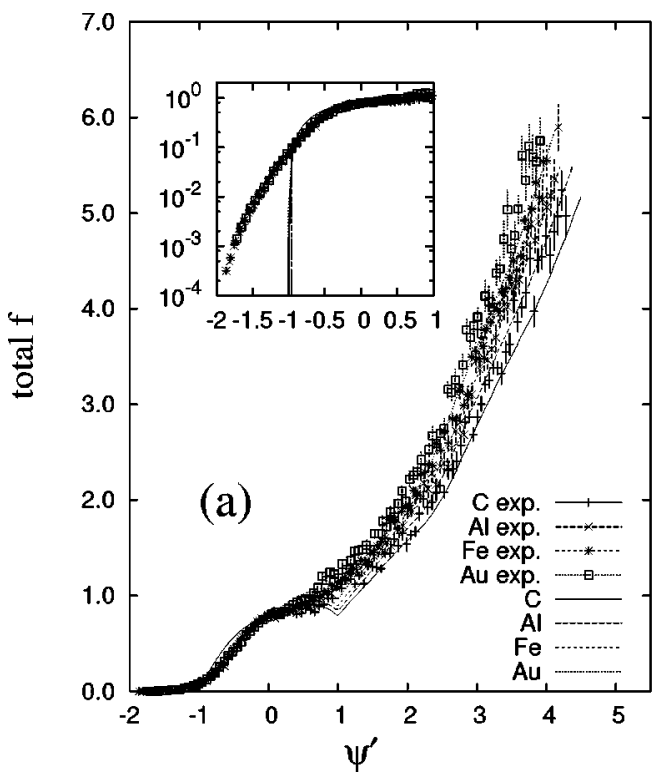

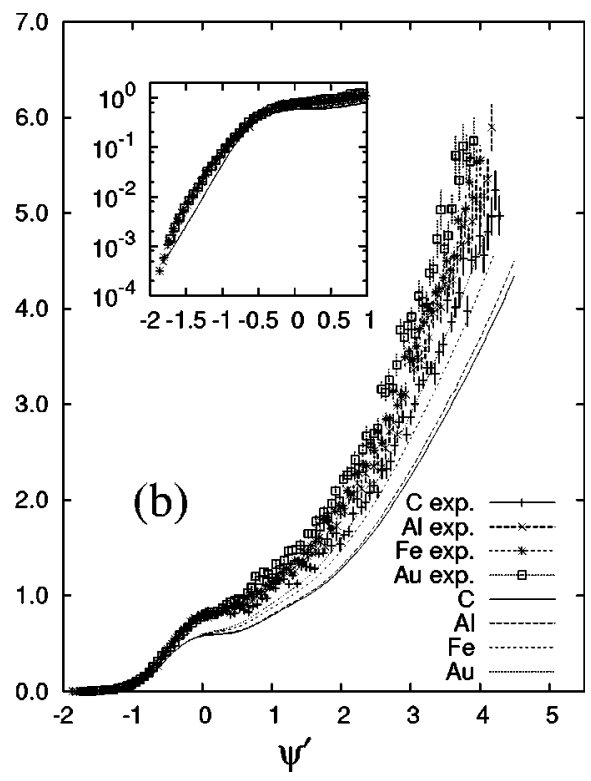

$035502-12$
FIG. 12. Total superscaling functions $f\left(\psi^{\prime}\right)$, as described in Sec. IV C, for the kinematical conditions $E_{e}=3.595 \mathrm{GeV}$ and $\theta_{e}$ $=16^{\circ}$. Theoretical results obtained within the RFG are shown in panel (a), while the ERFG case is presented in panel (b) 

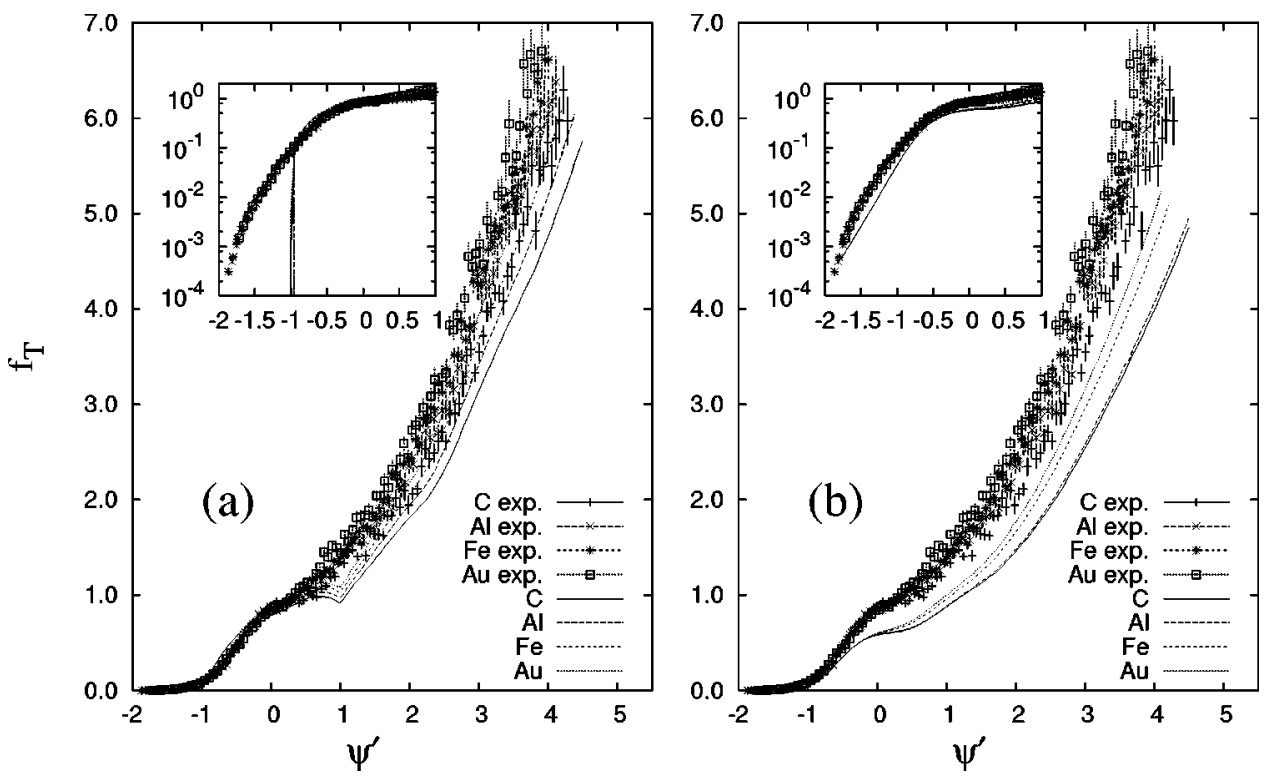

FIG. 13. As for Fig. 12, but showing the transverse superscaling functions $f_{T}\left(\psi^{\prime}\right)$ (see discussion in Sec. IV C). added to the nuclear model, going beyond the present onebody description, and that these must act mainly in the transverse channel. We have also checked that, in agreement with what previously observed, when $\theta$ increases the difference between total and transverse superscaling functions is more and more reduced and thus the disagreement between data and theory becomes the same for $f$ and $f_{T}$.

When examining the last two figures we see that the basic trend in the second-kind scaling behavior is present in the inelastic RFG modeling: for fixed kinematics the heavier nuclei with the larger values of $k_{F}$ have the higher responses at high inelasticity, and by roughly the right amount.

\section{CONCLUSIONS}

We have studied highly inelastic electron-nucleus scattering, from the resonance to the DIS region, in a unified relativistic framework. In particular we have calculated inclusive cross sections, response functions and scaling functions in the relativistic Fermi gas and in a phenomenological extension of it, named the ERFG, based on a fit of the scaling function in the quasielastic region. We have explored all high-quality experimental data available in the relevant highenergy domain, involving energy transfers from zero up to $\sim 3 \mathrm{GeV}$.

As discussed in detail in the results section the comparison between the data and the theoretical models is strongly dependent upon the kinematics. However, a few general features emerge from our analysis.

(1) In the quasielastic regime the RFG model approximately accounts for the experimental strength of the peak, but fails to reproduce the low- $\omega$ tail of the cross sections and predicts a pronounced unobserved "dip" to the right of the QEP. On the contrary, the ERFG model, while correctly reproducing cross sections at low-energy transfer, always underestimates the data around the peak.

(2) In the highly inelastic part of the spectrum the RFG roughly yields the experimental cross section for not too high-energy transfer (corresponding to smaller scattering angles) and overestimates the data when the inelasticity becomes very high (large scattering angles). In parallel, the ERFG underestimates the inelastic cross sections by $\sim 20 \%$ at small $\theta$, approaching the data as $\theta(\omega)$ increases.

(3) By analyzing the results in terms of the laboratory Bjorken scaling variable $x_{L}$ it is seen that the RFG works rather well at low $x_{L}(0.1-0.3)$, whereas the ERFG is more appropriate to describe the high- $x_{L}(\geqslant 0.6)$ data.

(4) A phenomenological energy shift is needed in both models to reproduce the QEP position, but it is irrelevant in the highly inelastic region. Moreover, concerning the separate responses, the longitudinal one appears to be more sensitive to the energy shift.

(5) The main impact of the nuclear medium on the responses and cross sections consists in washing out the resonance structure present in the single-nucleon responses as a consequence of the Fermi motion of nucleons inside the nucleus. In contrast, such an effect is negligible in the DIS regime.

The above findings point to the importance, in an intermediate region of energy transfers, of ingredients which are not included in the present approach, such as mesonexchange currents and correlations, in both $1 p-1 h$ and $2 p-2 h$ sectors. Preliminary results [22] seem to indicate that the $2 p-2 h$ MEC may play a crucial role in improving the agreement with the data, although a complete and consistent calculation of correlations and currents is still to be realized. A separate analysis of the longitudinal and transverse response functions (or, equivalently, of the $F_{1}$ and $F_{2}$ structure functions) based on the scaling approach shows that these missing contributions should be mostly active in the $T$ channel, thus supporting the relevance of meson-exchange currents.

Finally, it is interesting to note that the disagreement between ERFG predictions and the experimental results is not peculiar to the specific functional form of the phenomenological QE scaling function we have employed, but is essentially linked to its asymmetric shape. In fact, we checked that 
a simple "toy model" asymmetric scaling function (respecting of course the correct normalization) qualitatively yields similar results. We believe that the physical origin of this asymmetry is certainly worth further investigation.

\section{ACKNOWLEDGMENTS}

The authors wish to thank V. Barone for useful discussions on the DIS data, M. Ripani for providing the references and programs for the Bodek et al. fit of the inelastic structure functions, and I. Sick for providing data from experiments performed at SLAC and JLab. This work was partially supported by DGI (Spain), under Contract Nos. BFM200203315 and FPA2002-04181-C04-04, by the Junta de Andalucía and by the INFN-CICYT exchange program. The work of C.M. was supported in part by MEC (Spain) under Contract No. SB2000-0427. M.B.B. acknowledges financial support from MEC (Spain) for a sabbatical stay at University of Sevilla (Grant No. SAB2001-0025), during which part of this work was carried out. The work of T.W.D. was supported in part by funds provided by the U.S. Department of Energy under cooperative research Grant No. DE-FC0294ER40818.

APPENDIX A: INELASTIC TENSOR IN RFG

In this appendix we derive the general expression for $U^{\mu \nu}$ that enters in the hadronic inelastic tensor in Eq. (32). By using Eqs. (26) and (22) we can write the hadronic tensor as follows:

$$
\begin{aligned}
W_{\text {inel }}^{\mu \nu}(\kappa, \lambda)= & \frac{3 \mathcal{N} \tau}{2 \eta_{F}^{3} \kappa} \int_{\rho_{1}(\kappa, \lambda)}^{\rho_{2}(\kappa, \lambda)} d \rho \int_{0}^{2 \pi} \frac{d \Phi}{2 \pi} \int_{\epsilon_{0}(\rho)}^{\epsilon_{F}} d \bar{\epsilon}\left[-w_{1}(\tau, \rho)\right. \\
& \times\left(g^{\mu \nu}+\frac{\kappa^{\mu} \kappa^{\nu}}{\tau}\right)+w_{2}(\tau, \rho) \kappa^{\mu} \kappa^{\nu} \rho^{2} \\
& \left.+w_{2}(\tau, \rho) X^{\mu \nu}\right]
\end{aligned}
$$

having defined

$$
X^{\mu \nu}=\eta^{\mu} \eta^{\nu}+\rho\left(\eta^{\mu} \kappa^{\nu}+\eta^{\nu} \kappa^{\mu}\right) .
$$

To evaluate the above integral it is convenient to expand the four-vector $\eta^{\mu}$ (which is normalized to $\eta_{\mu} \eta^{\mu}=1$ ) in the basis $a^{\mu}=(\kappa, 0,0, \lambda), \quad \kappa^{\mu}=(\lambda, 0,0, \kappa), \quad t_{x}^{\mu}=(0,1,0,0), \quad t_{y}^{\mu}$ $=(0,0,1,0)$, namely,

$$
\eta^{\mu}=\eta_{k} \kappa^{\mu}+\eta_{a} a^{\mu}+\eta_{x} t_{x}^{\mu}+\eta_{y} t_{y}^{\mu}
$$

with

$$
\begin{gathered}
\eta_{k}=\eta \cos \theta_{0}=-\rho, \\
\eta_{a}=\frac{1}{\kappa}(\epsilon+\lambda \rho), \\
\eta_{x}=\eta \sin \theta_{0} \cos \Phi, \\
\eta_{y}=\eta \sin \theta_{0} \sin \Phi .
\end{gathered}
$$

The integral of the tensor in Eq. (A2) then becomes

$$
\begin{aligned}
\int_{0}^{2 \pi} \frac{d \Phi}{2 \pi} \int_{\epsilon_{0}(\rho)}^{\epsilon_{F}} d \bar{\epsilon} X^{\mu \nu}= & \int_{0}^{2 \pi} \frac{d \Phi}{2 \pi} \int_{\epsilon_{0}(\rho)}^{\epsilon_{F}} d \bar{\epsilon}\left[\eta_{k}^{2} \kappa^{\mu} \kappa^{\nu}+\eta_{a}^{2} a^{\mu} a^{\nu}\right. \\
& +\eta_{x}^{2} t_{x}^{\mu} t_{x}^{\nu}+\eta_{y}^{2} t_{y}^{\mu} t_{y}^{\nu}+\eta_{k} \eta_{a}\left(\kappa^{\mu} a^{\nu}+a^{\mu} \kappa^{\nu}\right) \\
& +\left(\eta_{\kappa} \kappa^{\mu}+\eta_{a} a^{\mu}\right) \kappa^{\nu} \rho \\
& \left.+\kappa^{\mu} \rho\left(\eta_{\kappa} \kappa^{\nu}+\eta_{a} a^{\nu}\right)\right] \\
= & \int_{0}^{2 \pi} \frac{d \Phi}{2 \pi} \int_{\epsilon_{0}(\rho)}^{\epsilon_{F}} d \bar{\epsilon}\left[-\rho^{2} \kappa^{\mu} \kappa^{\nu}+\eta_{a}^{2} a^{\mu} a^{\nu}\right. \\
& \left.+\eta_{x}^{2} t_{x}^{\mu} t_{x}^{\nu}+\eta_{y}^{2} t_{y}^{\mu} t_{y}^{\nu}\right],
\end{aligned}
$$

since

$$
\int_{0}^{2 \pi} d \Phi \eta_{x}=\int_{0}^{2 \pi} d \Phi \eta_{y}=\int_{0}^{2 \pi} d \Phi \eta_{x} \eta_{y}=0
$$

We now use the following integrals

$$
\begin{aligned}
\int_{0}^{2 \pi} \frac{d \Phi}{2 \pi} \int_{\epsilon_{0}(\rho)}^{\epsilon_{F}} d \bar{\epsilon} \eta_{x}^{2} & =\int_{0}^{2 \pi} \frac{d \Phi}{2 \pi} \int_{\epsilon_{0}(\rho)}^{\epsilon_{F}} d \bar{\epsilon} \eta_{y}^{2} \\
& =\frac{1}{2}\left(\epsilon_{F}-\epsilon_{0}\right) \theta\left(\epsilon_{F}-\epsilon_{0}\right) \mathcal{D}(\kappa, \tau, \rho),
\end{aligned}
$$

where $\mathcal{D}(\kappa, \tau, \rho)$ is given by Eq. (41), and

$$
\begin{aligned}
\int_{0}^{2 \pi} \frac{d \Phi}{2 \pi} \int_{\epsilon_{0}(\rho)}^{\epsilon_{F}} d \bar{\epsilon} \eta_{a}^{2}= & \int_{0}^{2 \pi} \frac{d \Phi}{2 \pi} \int_{\epsilon_{0}(\rho)}^{\epsilon_{F}} d \bar{\epsilon}_{\tau}^{-}\left(1+\tau \eta_{k}^{2}+\eta_{x}^{2}+\eta_{y}^{2}\right) \\
= & \left(\epsilon_{F}-\epsilon_{0}\right) \theta\left(\epsilon_{F}-\epsilon_{0}\right)\left[1+\tau \rho^{2}\right. \\
& \left.+\frac{3}{2} \mathcal{D}(\kappa, \tau, \rho)\right],
\end{aligned}
$$

and observe that

$$
t_{x}^{\mu} t_{x}^{\nu}+t_{y}^{\mu} t_{y}^{\nu}=-g^{\mu \nu}+\frac{a^{\mu} a^{\nu}}{\tau}-\frac{\kappa^{\mu} \kappa^{\nu}}{\tau} .
$$

By inserting the above relations into Eq. (A1) we get

$$
W_{\text {inel }}^{\mu \nu}(\kappa, \lambda)=\frac{3 \mathcal{N} \tau}{2 \eta_{F}^{3} \kappa} \int_{\rho_{1}(\kappa, \lambda)}^{\rho_{2}(\kappa, \lambda)} d \rho\left(\epsilon_{F}-\epsilon_{0}\right) \theta\left(\epsilon_{F}-\epsilon_{0}\right) U^{\mu \nu}(\kappa, \tau, \rho)
$$

with

$$
\begin{aligned}
U^{\mu \nu}(\kappa, \tau, \rho)= & -\left[w_{1}(\tau, \rho)+\frac{1}{2} w_{2}(\tau, \rho) \mathcal{D}(\kappa, \tau, \rho)\right] \\
& \times\left(g^{\mu \nu}+\frac{\kappa^{\mu} \kappa^{\nu}}{\tau}\right)+w_{2}(\tau, \rho)\left[1+\tau \rho^{2}\right. \\
& \left.+\frac{3}{2} \mathcal{D}(\kappa, \tau, \rho)\right] \frac{a^{\mu} a^{\nu}}{\tau} .
\end{aligned}
$$

From the above expression the longitudinal and transverse components in Eqs. (36) and (37) immediately follow.

The tensor in Eq. (A13) coincides with that in Eq. (32) if the scaling variable $\psi$ is introduced through the relation

$$
\epsilon_{F}-\epsilon_{0}=\xi_{F}\left(1-\psi_{X}\right)^{2}
$$




\section{APPENDIX B: INCLUSION OF THE ENERGY SHIFT}

In this appendix we derive explicit expressions for the QE and inelastic hadronic responses for the case in which a small energy shift is included in the analysis. As mentioned in Sec. $\mathrm{IV}$, to proceed one needs to assume a specific form for the single-nucleon tensors and the variable dependence of the single-nucleon structure functions. This choice is not unique and hence, some ambiguities enter in the analysis of the results. Here we adopted a specific strategy (see below); however, the cautionary statement should be made that other choices are possible and that these can lead to different results for the observables.

The specific form of the single-nucleon tensor we have selected is

$$
\begin{aligned}
w_{\text {inel }}^{\mu \nu}= & -w_{1}(\tau, \widetilde{\rho})\left(g^{\mu \nu}+\frac{\kappa^{\mu} \kappa^{\nu}}{\tau}\right)+w_{2}(\tau, \widetilde{\rho})\left(\eta^{\mu}+\frac{\eta \cdot \kappa}{\tau} \kappa^{\mu}\right) \\
& \times\left(\eta^{\nu}+\frac{\eta \cdot \kappa}{\tau} \kappa^{\nu}\right) .
\end{aligned}
$$

The longitudinal and transverse hadronic functions $U^{L, T}$ that result are

$$
\begin{gathered}
U^{L}=\frac{\kappa^{2}}{\tau}\left[\left(1+\tau \rho^{\prime 2}\right) w_{2}(\tau, \widetilde{\rho})-w_{1}(\tau, \widetilde{\rho})\right. \\
\left.+w_{2}(\tau, \widetilde{\rho}) \mathcal{D}_{L}^{\prime}\left(\kappa, \tau, \lambda_{\text {shift }}, \rho^{\prime}\right)\right], \\
U^{T}=2 w_{1}(\tau, \widetilde{\rho})+w_{2}(\tau, \widetilde{\rho}) \mathcal{D}_{T}^{\prime}\left(\kappa, \tau, \lambda_{\text {shift }}, \rho^{\prime}\right),
\end{gathered}
$$

where

$$
\begin{aligned}
\mathcal{D}_{T}^{\prime}\left(\kappa, \tau, \lambda_{\text {shift }}, \rho^{\prime}\right)= & \xi_{F}\left(1-\psi_{X}^{\prime 2}\right)\left[\frac{1}{\kappa} \sqrt{\tau^{\prime}\left(1+\tau^{\prime} \rho^{\prime 2}\right)}\right. \\
& \left.+\frac{1}{3} \xi_{F} \frac{\tau^{\prime}}{\kappa^{2}}\left(1+\psi_{X}^{\prime 2}\right)\right], \\
\mathcal{D}_{L}^{\prime}\left(\kappa, \tau, \lambda_{\text {shift }}, \rho^{\prime}\right)= & \frac{\tau}{\kappa^{2}}\left[\left(\frac{\lambda \tau^{\prime} \rho^{\prime}}{\tau}+1+\frac{\lambda}{\tau} \lambda_{\text {shift }}\right)^{2}\right. \\
& +\left(\frac{\lambda \tau^{\prime} \rho^{\prime}}{\tau}+1+\frac{\lambda}{\tau} \lambda_{\text {shift }}\right)\left(1+\frac{\lambda}{\tau} \lambda_{s h i f t}\right) \\
& \times \xi_{F}\left(1+\psi_{X}^{\prime 2}\right)+\left(1+\frac{\lambda}{\tau} \lambda_{s h i f t}\right)^{2} \\
& \left.\times \frac{1}{3} \xi_{F}^{2}\left(1+\psi_{X}^{\prime 2}+\psi_{X}^{\prime 4}\right)\right]-\left(1+\tau \rho^{\prime 2}\right)
\end{aligned}
$$

Notice that terms like $(\lambda / \tau) \lambda_{\text {shift }}$ and $\tau^{\prime} / \tau=1-\left(\lambda_{\text {shift }}^{2} / \tau\right)$ $+2(\lambda / \tau) \lambda_{\text {shift }}$, appearing in $\mathcal{D}_{L}^{\prime}$, can become large when $x_{L} \equiv \lambda / \tau$ is small, even if $\lambda_{\text {shift }}$ is small. We repeat that the above choice for the single-nucleon tensor is not unique, and that other choices involving the four-momentum $Q^{\prime \mu}$ instead of $Q^{\mu}$ are possible. Moreover, the arguments of the single-nucleon inelastic structure functions $w_{1,2}$, should be also considered carefully. As discussed in Sec. IV, here the inelasticity parameter, denoted as $\tilde{\rho}$, is given by

$$
\tilde{\rho} \equiv \frac{2 H \cdot Q^{\prime}}{\left|Q^{2}\right|}=\frac{W_{X}^{\prime 2}-m_{N}^{2}+\left|Q^{\prime 2}\right|}{\left|Q^{2}\right|}=\rho^{\prime} \frac{\tau^{\prime}}{\tau}
$$

which coincides with the expression given as $\left(2 m_{N} \widetilde{\omega}\right) /\left|Q^{2}\right|$, with $\widetilde{\omega}$ being the energy transferred to the nucleon in the system in which the nucleon is at rest. One should be aware that other alternatives exist, in particular, one can consider the inelasticity $\rho$ corresponding to a free nucleon at rest with final-state invariant mass equal to $W_{X}^{\prime}$.

The ambiguity introduced in the inelastic responses due to the energy shift is also present at the level of the hadronic QE response functions. Again, the problem is directly connected with the form assumed for the single-nucleon tensor. For consistency with the formalism used in the inelastic channel, in the QE process the single-nucleon tensor $w_{Q E}^{\mu \nu}$ is taken to be

$$
\begin{aligned}
w_{Q E}^{\mu \nu}= & -w_{1, Q E}(\tau)\left(g^{\mu \nu}+\frac{\kappa^{\mu} \kappa^{\nu}}{\tau}\right)+w_{2, Q E}(\tau)\left(\eta^{\mu}+\frac{\eta \cdot \kappa}{\tau} \kappa^{\mu}\right) \\
& \times\left(\eta^{\nu}+\frac{\eta \cdot \kappa}{\tau} \kappa^{\nu}\right) .
\end{aligned}
$$

The hadronic QE response functions within the RFG model are given by

$$
R_{Q E}^{L, T}=\frac{3 \mathcal{N}}{4 \eta_{F}^{3} \kappa m_{N}} \xi_{F}\left(1-\psi^{\prime 2}\right) \theta\left(1-\psi^{\prime 2}\right) U_{Q E}^{L, T}
$$

with the structure functions

$$
U_{Q E}^{L}=\frac{\kappa^{2}}{\tau}\left[(1+\tau) w_{2, e l}(\tau)-w_{1, e l}(\tau)+w_{2, e l}(\tau) \Delta_{L}^{\prime}\left(\tau, \kappa, \lambda_{\text {shift }}\right)\right]
$$

$$
U_{Q E}^{T}=2 w_{1, e l}(\tau)+w_{2, e l}(\tau) \Delta_{T}^{\prime}\left(\tau, \kappa, \lambda_{s h i f t}\right)
$$

The nuclear structure dependence is contained in the terms $\Delta_{L, T}^{\prime}$ in the form

$$
\begin{aligned}
\Delta_{T}^{\prime}\left(\kappa, \tau, \lambda_{\text {shift }}\right)= & \xi_{F}\left(1-\psi^{\prime 2}\right)\left[\frac{1}{\kappa} \sqrt{\tau^{\prime}\left(1+\tau^{\prime}\right)}+\frac{1}{3} \xi_{F} \frac{\tau^{\prime}}{\kappa^{2}}\left(1+\psi^{\prime 2}\right)\right] \\
\Delta_{L}^{\prime}\left(\kappa, \tau, \lambda_{\text {shift }}\right)= & \frac{\tau}{\kappa^{2}}\left[\left(\lambda \frac{\tau^{\prime}}{\tau}+1+\frac{\lambda}{\tau} \lambda_{\text {shift }}\right)^{2}\right. \\
& +\left(\lambda \frac{\tau^{\prime}}{\tau}+1+\frac{\lambda}{\tau} \lambda_{\text {shift }}\right)\left(1+\frac{\lambda}{\tau} \lambda_{\text {shift }}\right) \\
& \times \xi_{F}\left(1+\psi^{\prime 2}\right)+\left(1+\frac{\lambda}{\tau} \lambda_{\text {shift }}\right)^{2} \frac{1}{3} \xi_{F}^{2}\left(1+\psi^{\prime 2}\right. \\
& \left.\left.+\psi^{\prime \prime}\right)\right]-(1+\tau) .
\end{aligned}
$$

The scaling variable $\psi^{\prime}$ is given by 


$$
\psi^{\prime 2}=\frac{1}{\xi_{F}}\left(\kappa \sqrt{\frac{1}{\tau^{\prime}}+1}-\lambda^{\prime}-1\right)
$$

and the electromagnetic structure functions by

$$
w_{1, Q E}(\tau)=\tau G_{M}^{2}(\tau)
$$

$$
w_{2, Q E}(\tau)=\frac{G_{E}^{2}(\tau)+\tau G_{M}^{2}(\tau)}{1+\tau}
$$

with $G_{E, M}$ the proton or neutron Sachs electromagnetic form factors.

Finally, the "total" response functions are evaluated by adding the above QE responses to the inelastic ones, i.e., $R_{\text {tot }}^{L, T}=R_{Q E}^{L, T}+R_{\text {inel }}^{L, T}$
[1] W. M. Alberico, T. W. Donnelly, and A. Molinari, Nucl. Phys A512, 541 (1990).

[2] W. M. Alberico, M. B. Barbaro, A. De Pace, T. W. Donnelly, and A. Molinari, Nucl. Phys. A563, 605 (1993).

[3] M. B. Barbaro, A. De Pace, T. W. Donnelly, and A. Molinari, Nucl. Phys. A596, 553 (1996).

[4] J. E. Amaro, M. B. Barbaro, J. A. Caballero, T. W. Donnelly, and A. Molinari, Nucl. Phys. A643, 349 (1998).

[5] J. E. Amaro, M. B. Barbaro, J. A. Caballero, T. W. Donnelly, and A. Molinari, Nucl. Phys. A697, 388 (2002).

[6] J. E. Amaro, M. B. Barbaro, J. A. Caballero, T. W. Donnelly, and A. Molinari, Phys. Rep. 368, 317 (2002).

[7] J. E. Amaro, M. B. Barbaro, J. A. Caballero, T. W. Donnelly, and A. Molinari, Nucl. Phys. A723, 181 (2003).

[8] J. E. Amaro, M. B. Barbaro, J. A. Caballero, T. W. Donnelly, and A. Molinari, Nucl. Phys. A657, 161 (1999).

[9] L. Alvarez-Ruso, M. B. Barbaro, T. W. Donnelly, and A. Molinari, Nucl. Phys. A724, 157 (2003).

[10] B. D. Serot and J. D. Walecka, Adv. Nucl. Phys. 16, 1 (1986).

[11] W. M. Alberico, A. Molinari, T. W. Donnelly, E. L. Kronenberg, and J. W. Van Orden, Phys. Rev. C 38, 1801 (1988).

[12] R. P. Bickerstaff and A. W. Thomas, J. Phys. G 15, 1523 (1989).

[13] P. R. Norton, Rep. Prog. Phys. 66, 1253 (2003).

[14] S. Simula, Few-Body Syst., Suppl. 8, 423 (1995).

[15] A. Bodek and J. L. Ritchie, Phys. Rev. D 23, 1070 (1981); 24, 1400 (1981).

[16] T. W. Donnelly and I. Sick, Phys. Rev. Lett. 82, 3212 (1999).

[17] M. B. Barbaro, R. Cenni, A. De Pace, T. W. Donnelly, and A. Molinari, Nucl. Phys. A643, 137 (1998).

[18] T. W. Donnelly and I. Sick, Phys. Rev. C 60, 065502 (1999).

[19] D. B. Day, J. S. McCarthy, T. W. Donnelly, and I. Sick, Annu. Rev. Nucl. Part. Sci. 40, 357 (1990).

[20] C. Maieron, T. W. Donnelly, and I. Sick, Phys. Rev. C 65, 025502 (2002).

[21] J. Jourdan, Nucl. Phys. A603, 117 (1996).

[22] A. De Pace, M. Nardi, W. M. Alberico, T. W. Donnelly, and A. Molinari, Nucl. Phys. A726, 303 (2003).

[23] T. De Forest and J. D. Walecka, Adv. Phys. 15, 1 (1966).
[24] S. Boffi, C. Giusti, and F. D. Pacati, Phys. Rep. 226, 1 (1993).

[25] T. W. Donnelly, in Nuclear/Nucleonic Structure and Inclusive Electron Scattering, Proceedings of the International School of Physics "Enrico Fermi," Course CLIII, edited by A. Molinari and L. Riccati (IOS, Amsterdam, 2003), p. 183.

[26] J. D. Bjorken and S. D. Drell, Relativistic Quantum Mechanics (McGraw-Hill, New York, 1965).

[27] T. W. Donnelly and A. S. Raskin, Ann. Phys. (N.Y.) 169, 247 (1986).

[28] T. W. Donnelly, M. J. Musolf, W. M. Alberico, M. B. Barbaro, A. De Pace, and A. Molinari, Nucl. Phys. A541, 525 (1992).

[29] L. Alvarez-Ruso, M. B. Barbaro, T. W. Donnelly, and A. Molinari, Phys. Lett. B 497, 214 (2001).

[30] O. Benhar, V. R. Pandharipande, and I. Sick, Phys. Lett. B 410, 79 (1997).

[31] C. Ciofi Degli Atti and S. Liuti, Phys. Lett. B 225, 215 (1989).

[32] A. Bodek et al., Phys. Rev. D 20, 1471 (1979).

[33] A. Stein et al., Phys. Rev. D 12, 1884 (1975).

[34] P. Amaudruz et al., New Muon Collaboration, Phys. Lett. B 295, 159 (1992).

[35] L. W. Whitlow, S. Rock, A. Bodek, E. M. Riordan, and S. Dasu, Phys. Lett. B 250, 193 (1990).

[36] A. D. Martin, R. G. Roberts, W. J. Stirling, and R. S. Thorne, Eur. Phys. J. C 4, 463 (1998); see also http://durpdg.dur.ac.uk/ hepdata/mrs.html

[37] G. Hohler, E. Pietarinen, I. Sabba Stefanescu, F. Borkowski, G. G. Simon, V. H. Walther, and R. D. Wendling, Nucl. Phys. B114, 505 (1976).

[38] J. Arrington et al., Phys. Rev. Lett. 82, 2056 (1999).

[39] D. B. Day et al., Phys. Rev. C 48, 1849 (1993).

[40] R. G. Arnold et al., Phys. Rev. Lett. 52, 727 (1984).

[41] R. R. Whitney, I. Sick, J. R. Ficenec, R. D. Kephart, and W. P. Trower, Phys. Rev. C 9, 2230 (1974).

[42] S. Galster, H. Klein, J. Moritz, K. H. Schmidt, D. Wegener, and J. Bleckwenn, Nucl. Phys. B32, 221 (1971).

[43] F. Iachello, A. D. Jackson, and A. Lande, Phys. Lett. B 43, 191 (1973).

[44] P. E. Bosted, Phys. Rev. C 51, 409 (1995). 